\title{
4
}

\section{Theory of "Weak Value" and Quantum Mechanical Measurements}

\author{
Yutaka Shikano \\ Department of Physics, Tokyo Institute of Technology, Tokyo \\ Japan
}

\section{Introduction}

Quantum mechanics provides us many perspectives and insights on Nature and our daily life. However, its mathematical axiom initiated by von Neumann (121) is not satisfied to describe nature phenomena. For example, it is impossible not to explain a non self-adjoint operator, i.e., the momentum operator on a half line (See, e.g., Ref. (154).), as the physical observable. On considering foundations of quantum mechanics, the simple and specific expression is needed. One of the candidates is the weak value initiated by Yakir Aharonov and his colleagues (4). It is remarked that the idea of their seminal work is written in ref. (3). Furthermore, this quantity has a potentiality to explain the counter-factual phenomena, in which there is the contradiction under the classical logic, e.g., the Hardy paradox (64). If so, it may be possible to quantitatively explain quantum mechanics in the particle picture. In this review based on the author thesis (152), we consider the theory of the weak value and construct a measurement model to extract the weak value. See the other reviews in Refs. $(12 ; 14 ; 15 ; 20)$.

Let the weak value for an observable $A$ be defined as

$$
{ }_{f}\langle A\rangle_{i}^{w}:=\frac{\langle f|A| i\rangle}{\langle f \mid i\rangle}
$$

where $|i\rangle$ and $|f\rangle$ are called a pre- and post-selected state, respectively. As the naming of the "weak value", this quantity is experimentally accessible by the weak measurement as explained below. As seen in Fig. 1, the weak value can be measured as the shift of a meter of the probe after the weak interaction between the target and the probe with the specific post-selection of the target. Due to the weak interaction, the quantum state of the target is only slightly changed but the information of the desired observable $A$ is encoded in the probe by the post-selection. While the previous studies of the weak value since the seminal paper (4), which will be reviewed in Sec. 3, are based on the measurement scheme, there are few works that the weak value is focused on and is independent of the measurement scheme. Furthermore, in these 20 years, we have not yet understood the mathematical properties of the weak value. In this chapter, we review the historical backgrounds of the weak value and the weak measurement and recent development on the measurement model to extract the weak value.

\section{Review of quantum operation}

The time evolution for the quantum state and the operation for the measurement are called a quantum operation. In this section, we review a general description of the quantum operation. Therefore, the quantum operation can describe the time evolution for the quantum state, the 


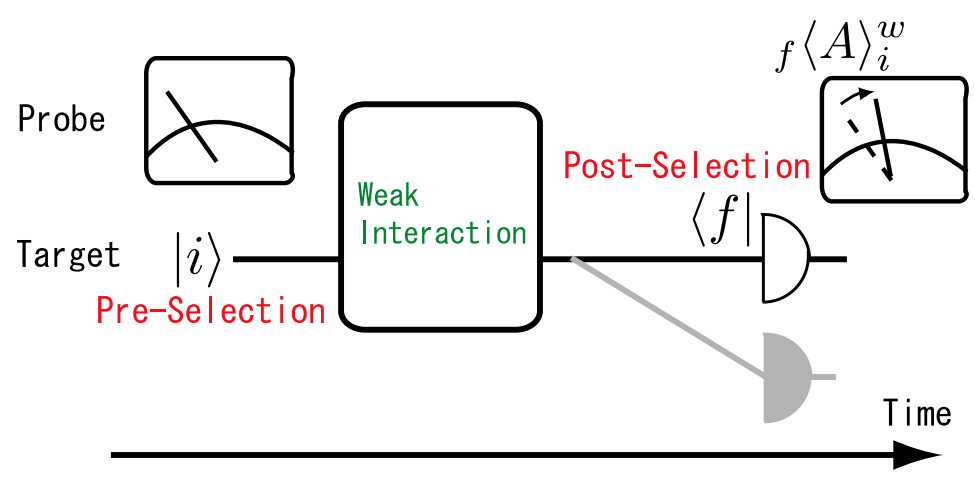

Fig. 1. Schematic figure of the weak measurement.

control of the quantum state, the quantum measurement, and the noisy quantum system in the same formulation.

\subsection{Historical remarks}

Within the mathematical postulates of quantum mechanics (121), the state change is subject to the Schrödinger equation. However, the state change on the measurement is not subject to this but is subject to another axiom, conventionally, von Neumann-Lüders projection postulate (105). See more details on quantum measurement theory in the books $(31 ; 40 ; 194)$. Let us consider a state change from the initial state $|\psi\rangle$ on the projective measurement ${ }^{1}$ for the operator $A=\sum_{j} a_{j}\left|a_{j}\right\rangle\left\langle a_{j}\right|$. From the Born rule, the probability to obtain the measurement outcome, that is, the eigenvalue of the observable $A$, is given by

$$
\operatorname{Pr}\left[A=a_{m}\right]=\left|\left\langle a_{m} \mid \psi\right\rangle\right|^{2}=\operatorname{Tr}\left[|\psi\rangle\left\langle\psi|\cdot| a_{m}\right\rangle\left\langle a_{m}\right|\right]=\operatorname{Tr} \rho P_{a_{m}},
$$

where $\rho:=|\psi\rangle\langle\psi|$ and $P_{a_{m}}=\left|a_{m}\right\rangle\left\langle a_{m}\right|$. After the measurement with the measurement outcome $a_{m}$, the quantum state change is given by

$$
|\psi\rangle \rightarrow\left|a_{m}\right\rangle,
$$

which is often called the "collapse of wavefunction" or "state reduction". This implies that it is necessary to consider the non-unitary process even in the isolated system. To understand the measuring process as quantum dynamics, we need consider the general theory of quantum operations.

\subsection{Operator-sum representation}

Let us recapitulate the general theory of quantum operations of a finite dimensional quantum system (122). All physically realizable quantum operations can be generally described by a completely positive $(\mathrm{CP})$ map $(127 ; 128)$, since the isolated system of a target system and an auxiliary system always undergoes the unitary evolution according to the axiom of quantum mechanics (121). Physically speaking, the operation of the target system should be described as a positive map, that is, the map from the positive operator to the positive operator, since the density operator is positive. Furthermore, if any auxiliary system is coupled to the target one,

\footnotetext{
${ }^{1}$ This measurement is often called the von Neumann measurement or the strong measurement.
} 
the quantum dynamics in the compound system should be also described as the positive map since the compound system should be subject to quantum mechanics. Given the positive map, the positive map is called a CP map if and only if the positive map is also in the compound system coupled to any auxiliary system. One of the important aspects of the CP map is that all physically realizable quantum operations can be described only by operators defined in the target system. Furthermore, the auxiliary system can be environmental system, the probe system, and the controlled system. Regardless to the role of the auxiliary system, the CP map gives the same description for the target system. On the other hand, both quantum measurement and decoherence give the same role for the target system.

Let $\mathcal{E}$ be a positive map from $\mathcal{L}\left(\mathcal{H}_{s}\right)$, a set of linear operations on the Hilbert space $\mathcal{H}_{s}$, to $\mathcal{L}\left(\mathcal{H}_{s}\right)$. If $\mathcal{E}$ is completely positive, its trivial extension $\mathcal{K}$ from $\mathcal{L}\left(\mathcal{H}_{s}\right)$ to $\mathcal{L}\left(\mathcal{H}_{s} \otimes \mathcal{H}_{e}\right)$ is also positive such that

$$
\mathcal{K}(|\alpha\rangle):=(\mathcal{E} \otimes \mathbf{1})(|\alpha\rangle\langle\alpha|)>0,
$$

for an arbitrary state $|\alpha\rangle \in \mathcal{H}_{s} \otimes \mathcal{H}_{p}$, where $\mathbf{1}$ is the identity operator. We assume without loss of generality $\operatorname{dim} \mathcal{H}_{s}=\operatorname{dim} \mathcal{H}_{e}<\infty$. Throughout this chapter, we concentrate on the case that the target state is pure though the generalization to mixed states is straightforward. From the complete positivity, we obtain the following theorem for quantum state changes.

Theorem 2.1. Let $\mathcal{E}$ be a $C P$ map from $\mathcal{H}_{s}$ to $\mathcal{H}_{s}$. For any quantum state $|\psi\rangle_{s} \in \mathcal{H}_{s}$, there exist a map $\sigma$ and a pure state $|\alpha\rangle \in \mathcal{H}_{s} \otimes \mathcal{H}_{e}$ such that

$$
\mathcal{E}\left(|\psi\rangle_{s}\langle\psi|\right)=e_{e}\langle\tilde{\psi}| \mathcal{K}(|\alpha\rangle)|\tilde{\psi}\rangle_{e},
$$

where

$$
|\psi\rangle_{s}=\sum_{k} \psi_{k}|k\rangle_{s}, \quad|\tilde{\psi}\rangle_{e}=\sum_{k} \psi_{k}^{*}|k\rangle_{e},
$$

which represents the state change for the density operator.

Proof. We can write in the Schmidt form as

$$
|\alpha\rangle=\sum_{m}|m\rangle_{s}|m\rangle_{e} .
$$

We rewrite the right hand sides of Eq. (5) as

$$
\begin{aligned}
\mathcal{K}(|\alpha\rangle) & =(\mathcal{E} \otimes \mathbf{1})\left(\sum_{m, n}|m\rangle_{s}|m\rangle_{e s}\left\langle\left. n\right|_{e}\langle n|\right)\right. \\
& =\sum_{m, n}|m\rangle_{e}\langle n| \mathcal{E}\left(|m\rangle_{s}\langle n|\right)
\end{aligned}
$$

to obtain

$$
{ }_{e}\langle m| \mathcal{K}(|\alpha\rangle)|n\rangle_{e}=\mathcal{E}\left(|m\rangle_{s}\langle n|\right) .
$$

By linearity, the desired equation (5) can be derived.

From the complete positivity, $\mathcal{K}(|\alpha\rangle)>0$ for all $|\alpha\rangle \in \mathcal{H}_{s} \otimes \mathcal{H}_{e}$, we can express $\sigma(|\alpha\rangle)$ as

$$
\mathcal{K}(|\alpha\rangle)=\sum_{m} s_{m}\left|\hat{s}_{m}\right\rangle\left\langle\hat{s}_{m}\left|=\sum_{m}\right| s_{m}\right\rangle\left\langle s_{m}\right|,
$$


where $s_{m}$ 's are positive and $\left\{\left|\hat{s}_{m}\right\rangle\right\}$ is a complete orthonormal set with $\left|s_{m}\right\rangle:=\sqrt{s_{m}}\left|\hat{s}_{m}\right\rangle$. We define the Kraus operator $E_{m}(95)$ as

$$
E_{m}|\psi\rangle_{s}:=e\left\langle\tilde{\psi} \mid s_{m}\right\rangle .
$$

Then, the quantum state change becomes the operator-sum representation,

$$
\sum_{m} E_{m}|\psi\rangle_{s}\left\langle\psi\left|E_{m}^{\dagger}=\sum_{m}\left\langle\tilde{\psi} \mid s_{m}\right\rangle\left\langle s_{m} \mid \tilde{\psi}\right\rangle_{e}={ }_{e}\langle\tilde{\psi}| \mathcal{K}(|\alpha\rangle)\right| \tilde{\psi}\right\rangle_{e}=\mathcal{E}\left(|\psi\rangle_{s}\langle\psi|\right)
$$

It is emphasized that the quantum state change is described solely in terms of the quantities of the target system.

\subsection{Indirect quantum measurement}

In the following, the operator-sum representation of the quantum state change is related to the indirect measurement model. Consider the observable $A_{s}$ and $B_{p}$ for the target and probe systems given by

$$
A_{s}=\sum_{j} a_{j}\left|a_{j}\right\rangle_{s}\left\langle a_{j}\left|, \quad B_{p}=\sum_{j} b_{j}\right| b_{j}\right\rangle_{p}\left\langle b_{j}\right|,
$$

respectively. We assume that the interaction Hamiltonian is given by

$$
H_{\text {int }}(t)=g\left(A_{s} \otimes B_{p}\right) \delta\left(t-t_{0}\right),
$$

where $t_{0}$ is measurement time. Here, without loss of generality, the interaction is impulsive and the coupling constant $g$ is scalar. The quantum dynamics for the compound system is given by

$$
\left|s_{m}\right\rangle\left\langle s_{m}\right|=U\left(|\psi\rangle_{s}\langle\psi|\otimes| \phi\rangle_{p}\langle\phi|\right) U^{\dagger},
$$

where $|\psi\rangle_{s}$ and $|\phi\rangle_{p}$ are the initial quantum state on the target and probe systems, respectively. For the probe system, we perform the projective measurement for the observable $B_{p}$. The probability to obtain the measurement outcome $b_{m}$ is given by

$$
\begin{aligned}
\operatorname{Pr}\left[B_{p}=b_{m}\right] & =\operatorname{Tr}_{s}\left\langle b_{m}\left|U\left(|\psi\rangle_{s}\langle\psi|\otimes| \phi\rangle_{p}\langle\phi|\right) U^{\dagger}\right| b_{m}\right\rangle, \\
& =\operatorname{Tr}_{s} E_{m}|\psi\rangle_{s}\left\langle\psi\left|E_{m}^{\dagger}=\operatorname{Tr}_{s}\right| \psi\right\rangle_{s}\langle\psi| M_{m},
\end{aligned}
$$

where the Kraus operator $E_{m}$ is defined as

$$
E_{m}:={ }_{p}\left\langle b_{m}|U| \phi\right\rangle_{p},
$$

and $M_{m}:=E_{m}^{\dagger} E_{m}$ is called a positive operator valued measure (POVM) (45). The POVM has the same role of the spectrum of the operator $A_{S}$ in the case of the projective measurement. To derive the projective measurement from the indirect measurement, we set the spectrum of the operator $A_{s}$ as the POVM, that is, $M_{m}=\left|a_{m}\right\rangle_{s}\left\langle a_{m}\right|$. Since the sum of the probability distribution over the measurement outcome equals to one, we obtain

$$
\begin{aligned}
\sum_{m} \operatorname{Pr}\left[B_{p}=b_{m}\right]=1 & \Longleftrightarrow \sum_{m} \operatorname{Tr}|\psi\rangle_{s}\left\langle\psi\left|M_{m}=\operatorname{Tr}\right| \psi\right\rangle_{s}\langle\psi| \sum_{m} M_{m}=1 \\
& \rightarrow \sum_{m} M_{m}=\mathbf{1} .
\end{aligned}
$$

Here, the last line uses the property of the density operator, $\operatorname{Tr}|\psi\rangle_{s}\langle\psi|=1$ for any $|\psi\rangle$. 


\section{Review of weak value}

In Secs. 2.1 and 2.3, the direct and indirect quantum measurement schemes, we only get the probability distribution. However, the probability distribution is not the only thing that is experimentally accessible in quantum mechanics. In quantum mechanics, the phase is also an essential ingredient and in particular the geometric phase is a notable example of an experimentally accessible quantity (150). The general experimentally accessible quantity which contains complete information of the probability and the phase seems to be the weak value advocated by Aharonov and his collaborators $(4 ; 14)$. They proposed a model of weakly coupled system and probe, see Sec. 4.3, to obtain information to a physical quantity as a "weak value" only slightly disturbing the state. Here, we briefly review the formal aspects of the weak value.

For an observable $A$, the weak value $\langle A\rangle_{w}$ is defined as

$$
\langle A\rangle_{w}:=\frac{\left\langle f\left|U\left(t_{f}, t\right) A U\left(t, t_{i}\right)\right| i\right\rangle}{\left\langle f\left|U\left(t_{f}, t_{i}\right)\right| i\right\rangle} \in \mathbb{C},
$$

where $|i\rangle$ and $\langle f|$ are normalized pre-selected ket and post-selected bra state vectors, respectively (4). Here, $U\left(t_{2}, t_{1}\right)$ is an evolution operator from the time $t_{1}$ to $t_{2}$. The weak value $\langle A\rangle_{w}$ actually depends on the pre- and post-selected states $|i\rangle$ and $\langle f|$ but we omit them for notational simplicity in the case that we fix them. Otherwise, we write them explicitly as ${ }_{f}\langle A\rangle_{i}^{w}$ instead for $\langle A\rangle_{w}$. The denominator is assumed to be non-vanishing. This quantity is, in general, in the complex number $\mathbb{C}$. Historically, the terminology "weak value" comes from the weak measurement, where the coupling between the target system and the probe is weak, explained in the following section. Apart from their original concept of the weak value and the weak measurement, we emphasize that the concept of the weak value is independent of the weak measurement ${ }^{2}$. To take the weak value as a priori given quantity in quantum mechanics, we will construct the observable-independent probability space. In the conventional quantum measurement theory, the probability space, more precisely speaking, the probability measure, depends on the observable (151, Sec. 4.1) ${ }^{3}$.

Let us calculate the expectation value in quantum mechanics for the quantum state $|\psi\rangle$ as

$$
\begin{aligned}
\operatorname{Ex}[A]=\langle\psi|A| \psi\rangle & =\int d \phi\langle\psi \mid \phi\rangle\langle\phi|A| \psi\rangle=\int d \phi\langle\psi \mid \phi\rangle \cdot\langle\phi \mid \psi\rangle \frac{\langle\phi|A| \psi\rangle}{\langle\phi \mid \psi\rangle}, \\
& =\int d \phi|\langle\psi \mid \phi\rangle|^{2}{ }_{\phi}\langle A\rangle_{\psi^{\prime}}^{w}
\end{aligned}
$$

where $h_{A}[|\phi\rangle]={ }_{\phi}\langle A\rangle_{\psi}^{w}$ is complex random variable and $d P:=|\langle\phi \mid \psi\rangle|^{2} d \phi$ is the probability measure and is independent of the observable $A$. Therefore, the event space $\Omega=\{|\phi\rangle\}$ is taken as the set of the post-selected state. This formula means that the extended probability theory corresponds to the Born rule. From the conventional definition of the variance in

\footnotetext{
2 This concept is shared in Refs. $(1 ; 49 ; 51 ; 78 ; 81 ; 82 ; 117 ; 130)$.

${ }^{3}$ Due to this, the probability in quantum mechanics cannot be applied to the standard probability theory. As another approach to resolve this, there is the quantum probability theory (138).
} 
quantum mechanics, we obtain the variance as

$$
\begin{aligned}
\operatorname{Var}[A] & =\int \mid\left. h_{A}[|\phi\rangle]\right|^{2} d P-\left(\int h_{A}[|\phi\rangle] d P\right)^{2} \\
& =\int\left|\frac{\langle\phi|A| \psi\rangle}{\langle\phi \mid \psi\rangle}\right|^{2}|\langle\phi \mid \psi\rangle|^{2} d \phi-\left(\int \frac{\langle\phi|A| \psi\rangle}{\langle\phi \mid \psi\rangle}|\langle\phi \mid \psi\rangle|^{2} d \phi\right)^{2} \\
& =\int|\langle\phi|A| \psi\rangle|^{2} d \phi-\left(\int\langle\psi \mid \phi\rangle\langle\phi|A| \psi\rangle d \phi\right)^{2} \\
& =\int\langle\psi|A| \phi\rangle\langle\phi|A| \psi\rangle d \phi-(\langle\psi|A| \psi\rangle)^{2} \\
& =\left\langle\psi\left|A^{2}\right| \psi\right\rangle-(\langle\psi|A| \psi\rangle)^{2} .
\end{aligned}
$$

This means that the observable-independent probability space can be characterized by the weak value (155). From another viewpoint of the weak value, the statistical average of the weak value coincides with the expectation value in quantum mechanics (7). This can be interpreted as the probability while this allows the "negative probability" 4 . On this idea, the uncertainty relationship was analyzed on the Robertson inequality $(58 ; 163)$ and on the Ozawa inequality (106), which the uncertainty relationships are reviewed in Ref. (151, Appendix A). Also, the joint probability for the compound system was analyzed in Refs. $(27 ; 30)$. Furthermore, if the operator $A$ is a projection operator $A=|a\rangle\langle a|$, the above identity becomes an analog of the Bayesian formula,

$$
|\langle a \mid \psi\rangle|^{2}=\int_{\phi}\langle\mid a\rangle\langle a \mid\rangle_{\psi}^{w}|\langle\phi \mid \psi\rangle|^{2} d \phi .
$$

The left hand side is the probability to obtain the state $|a\rangle$ given the initial state $|\psi\rangle$. From this, one may get some intuition by interpreting the weak value ${ }_{\phi}\langle\mid a\rangle\langle a \mid\rangle_{\psi}^{w}$ as the complex conditional probability of obtaining the result $|a\rangle$ under an initial condition $|i\rangle$ and a final condition $|f\rangle$ in the process $|i\rangle \rightarrow|a\rangle \rightarrow|f\rangle(170 ; 171)^{5}$. Of course, we should not take the strange weak values too literally but the remarkable consistency of the framework of the weak values due to Eq. (21) and a consequence of the completeness relation,

$$
\sum_{a}\langle\mid a\rangle\langle a \mid\rangle_{w}=1
$$

may give a useful concept to further push theoretical consideration by intuition.

This interpretation of the weak values gives many possible examples of strange phenomena like a negative kinetic energy (11), a spin $100 \hbar$ for an electron $(4 ; 23 ; 52 ; 60)$ and a superluminal propagation of light $(142 ; 162)$ and neutrino $(28 ; 176)$ motivated by the

\footnotetext{
${ }^{4}$ The concept of negative probability is not new, e.g., see Refs. $(47 ; 57 ; 65 ; 66 ; 71)$. The weak value defined by Eq. (18) is normally called the transition amplitude from the state $|\psi\rangle$ to $\langle\phi|$ via the intermediate state $|a\rangle$ for $A=|a\rangle\langle a|$, the absolute value squared of which is the probability for the process. But the three references quoted above seem to suggest that they might be interpreted as probabilities in the case that the process is counter-factual, i.e., the case that the intermediate state $|a\rangle$ is not projectively measured. The description of intermediate state $|a\rangle$ in the present work is counter-factual or virtual in the sense that the intermediate state would not be observed by projective measurements. Feynman's example is the counter-factual "probability" for an electron to have its spin up in the $x$-direction and also spin down in the $z$-direction (57).

${ }^{5}$ The interpretation of the weak value as a complex probability is suggested in the literature (118).
} 
OPERA experiment (125). The framework of weak values has been theoretically applied to foundations of quantum physics, e.g., the derivation of the Born rule from the alternative assumption for a priori measured value (74), the relationship to the uncertainty relationship (72), the quantum stochastic process (190), the tunneling traverse time (135; $170 ; 171)$, arrival time and time operator $(21 ; 39 ; 146 ; 147)$, the decay law $(46 ; 187)$, the non-locality $(32 ; 180 ; 181)$, especially, quantum non-locality, which is characterized by the modular variable, consistent history $(87 ; 188)$, Bohmian quantum mechanics (98), semi-classical weak values on the tunneling (175), the quantum trajectory (192), and classical stochastic theory (177). Also, in quantum information science, the weak value was analyzed on quantum computation $(35 ; 126)$, quantum communications $(29 ; 36)$, quantum estimation, e.g., state tomography $(67-69 ; 111 ; 158)$ and the parameter estimation $(70 ; 73 ; 157)$, the entanglement concentration (113), the quasi-probability distribution $(24 ; 61 ; 148 ; 183)$ and the cloning of the unknown quantum state with hint (161). Furthermore, this was applied to the cosmological situations in quantum-mechanical region, e.g., the causality (22), the inflation theory (42), backaction of the Hawking radiation from the black hole $(34 ; 54 ; 55)$, and the new interpretation of the universe $(9 ; 53 ; 62)$. However, the most important fact is that the weak value is experimentally accessible so that the intuitive argument based on the weak values can be either verified or falsified by experiments. There are many experimental proposals to obtain the weak value in the optical $(2 ; 44 ; 88 ; 101 ; 112 ; 159 ; 197)$ and the solid-state $(83 ; 84 ; 94 ; 115 ; 143 ; 144 ; 191 ; 200)$ systems. Recently, the unified viewpoint was found in the weak measurement (92).

On the realized experiments on the weak value, we can classify the three concepts: (i) testing the quantum theory, (ii) the amplification of the tiny effect in quantum mechanics, and (iii) the quantum phase.

(i) Testing the quantum theory. The weak value can solve many quantum paradoxes seen in the book (14). The Hardy paradox (64), which there occurs in two Mach-Zehnder interferometers of the electron and the position, was resolved by the weak value (8) and was analyzed deeper (75). This paradoxical situation was experimentally demonstrated in the optical setup $(107 ; 198)$. By the interference by the polarization (131) and shifting the optical axis (141), the spin beyond the eigenvalue is verified. By the latter technique, the three-box paradox $(16 ; 188)$ was realized $(139)$. Thereafter, the theoretical progresses are the contextuality on quantum mechanics (178), the generalized N-box paradox (99), and the relationship to the Kirkpatrick game (137). The weak value is used to show the violation of the Leggett-Garg inequality $(110 ; 191)$. This experimental realizations were demonstrated in the system of the superconducting qubit (97), the optical systems $(50 ; 134)$. Furthermore, since the weak value for the position observable $|x\rangle\langle x|$ with the pre-selected state $|\psi\rangle$ and the post-selection $|p\rangle$ is given by

$$
\langle\mid x\rangle\langle x \mid\rangle_{w}=\frac{\langle p \mid x\rangle\langle x \mid \psi\rangle}{\langle p \mid \psi\rangle}=\frac{e^{i x p} \psi(x)}{\phi(p)},
$$

we obtain the wavefunction $\psi(x):=\langle x \mid \psi\rangle$ as the weak value with the multiplication factor $1 / \phi(0)$ with $\phi(p):=\langle p \mid \psi\rangle$ in the case of $p=0$. Using the photon transverse wavefunction, there are experimentally demonstrated by replacing the weak measurement for the position as the polarization measurement (109). This paper was theoretically criticized to compare the standard quantum state tomography for the phase space in Ref. (63) and was generalized to a conventionally unobservable (108). As other examples, there are the detection of the superluminal signal (37), the quantum non-locality (165), and the Bohmian trajectory $(91 ; 149)$ on the base of the theoretical analysis $(193)$. 
(ii) Amplification of the tiny effect in quantum mechanics. Since the weak value has the denominator, the weak value is very large when the pre- and post-selected states are almost orthogonal $^{6}$. This is practical advantage to use the weak value. While the spin Hall effect of light (124) is too tiny effect to observe its shift in the conventional scheme, by almost orthogonal polarizations for the input and output, this effect was experimentally verified (76) to be theoretically analyzed from the viewpoint of the spin moments (96). Also, some interferometers were applied. The beam deflection on the Sagnac interferometer (48) was shown to be supported by the classical and quantum theoretical analyses $(77){ }^{7}$. Thereafter, optimizing the signal-to-noise ratio $(166 ; 184)$, the phase amplification $(168 ; 169)$, and the precise frequency measurement (167) were demonstrated. As another example, there is shaping the laser pulse beyond the diffraction limit (136). According to Steinberg (172), in his group, the amplification on the single-photon nonlinearity has been progressed to be based on the theoretical proposal (56). While the charge sensing amplification was proposed in the solid-state system (200), there is no experimental demonstration on the amplification for the solid-state system. Furthermore, the upper bound of the amplification has not yet solved. Practically, this open problem is so important to understand the relationship to the weak measurement regime.

(iii) Quantum phase. The argument of the weak value for the projection operator is the geometric phase as

$$
\begin{aligned}
\gamma & :=\arg \left\langle\psi_{1} \mid \psi_{2}\right\rangle\left\langle\psi_{2} \mid \psi_{3}\right\rangle\left\langle\psi_{3} \mid \psi_{1}\right\rangle \\
& =\arg \frac{\left\langle\psi_{1} \mid \psi_{2}\right\rangle\left\langle\psi_{2} \mid \psi_{3}\right\rangle\left\langle\psi_{3} \mid \psi_{1}\right\rangle}{\left|\left\langle\psi_{3} \mid \psi_{1}\right\rangle\right|^{2}}=\arg \frac{\left\langle\psi_{1} \mid \psi_{2}\right\rangle\left\langle\psi_{2} \mid \psi_{3}\right\rangle}{\left\langle\psi_{1} \mid \psi_{3}\right\rangle} \\
& =\arg \psi_{1}\left\langle\mid \psi_{2}\right\rangle\left\langle\psi_{2} \mid\right\rangle_{\psi_{3}}^{w} .
\end{aligned}
$$

where the quantum states, $\left|\psi_{1}\right\rangle,\left|\psi_{2}\right\rangle$, and $\left|\psi_{3}\right\rangle$, are the pure states (160). Here, the quantum states, $\left|\psi_{1}\right\rangle$ and $\left|\psi_{3}\right\rangle$, are the post- and pre-selected states, respectively. Therefore, we can evaluate the weak value from the phase shift (174). Of course, vice versa (38). Tamate et al. proposal was demonstrated on the relationship to quantum eraser (90) and by the a three-pinhole interferometer (89). The phase shift from the zero mode to $\pi$ mode was observed by using the interferometer with a Cs vapor (41) and the phase shift in the which-way path experiment was demonstrated (116). Furthermore, by the photonic crystal, phase singularity was demonstrated (164).

(iv) Miscellaneous. The backaction of the weak measurement is experimentally realized in the optical system (79). Also, the parameter estimation using the weak value is demonstrated (73).

\section{Historical background - two-state vector formalism}

In this section, we review the original concept of the two-state vector formalism. This theory is seen in the reviewed papers $(15 ; 20)$.

\footnotetext{
${ }^{6}$ Unfortunately, the signal to noise ratio is not drastically changed under the assumption that the probe wavefunction is Gaussian on a one-dimensional parameter space.

${ }^{7}$ Unfortunately, the experimental data are mismatched to the theoretical prediction. While the authors claimed that this differences results from the stray of light, the full-order calculation even is not mismatched (93). However, this difference remains the open problem.
} 


\subsection{Time symmetric quantum measurement}

While the fundamental equations of the microscopic physics are time symmetric, for example, the Newton equation, the Maxwell equation, and the Schrödinger equation ${ }^{8}$, the quantum measurement is not time symmetric. This is because the quantum state after quantum measurement depends on the measurement outcome seen in Sec. 2. The fundamental equations of the microscopic physics can be solved to give the initial boundary condition. To construct the time symmetric quantum measurement, the two boundary conditions, which is called pre- and post-selected states, are needed. The concept of the pre- and post-selected states is called the two-state vector formalism (6). In the following, we review the original motivation to construct the time symmetric quantum measurement.

Let us consider the projective measurement for the observable $A=\sum_{i} a_{i}\left|a_{i}\right\rangle\left\langle a_{i}\right|$ with the initial boundary condition denoted as $|i\rangle$ at time $t_{i}$. To take quantum measurement at time $t_{0}$, the probability to obtain the measurement outcome $a_{j}$ is given by

$$
\operatorname{Pr}\left[A=a_{j}\right]=\left\|\left\langle a_{j}|U| i\right\rangle\right\|^{2},
$$

with the time evolution $U:=U\left(t_{0}, t_{i}\right)$. After the projective measurement, the quantum state becomes $\left|a_{j}\right\rangle$. Thereafter, the quantum state at $t_{f}$ is given by $\left|\varphi_{j}\right\rangle:=V\left|a_{j}\right\rangle$ with $V=U\left(t_{f}, t_{0}\right)$. the probability to obtain the measurement outcome $a_{j}$ can be rewritten as

$$
\operatorname{Pr}\left[A=a_{j}\right]=\frac{\left\|\left\langle\varphi_{j}|V| a_{j}\right\rangle\right\|^{2}\left\|\left\langle a_{j}|U| i\right\rangle\right\|^{2}}{\sum_{j}\left\|\left\langle\varphi_{j}|V| a_{j}\right\rangle\right\|^{2}\left\|\left\langle a_{j}|U| i\right\rangle\right\|^{2}} .
$$

It is noted that $\left\|\left\langle\varphi_{j}|V| a_{j}\right\rangle\right\|^{2}=1$. Here, we consider the backward time evolution from the quantum state $\left|\varphi_{j}\right\rangle$ at time $t_{f}$. We always obtain the quantum state $\left|a_{j}\right\rangle$ after the projective measurement at time $t_{0}$. Therefore, the quantum state at time $t_{i}$ is given by

$$
|\tilde{i}\rangle:=U^{\dagger}\left|a_{j}\right\rangle\left\langle a_{j}\left|V^{\dagger}\right| \varphi_{j}\right\rangle=U^{\dagger}\left|a_{j}\right\rangle .
$$

In general, $|\tilde{i}\rangle$ is different from $|i\rangle$. Therefore, projective measurement is time asymmetric.

To construct the time-symmetric quantum measurement, we add the boundary condition at time $t_{f}$. Substituting the quantum state $\left|\varphi_{j}\right\rangle$ to the specific one denoted as $|f\rangle$, which is called the post-selected state, the probability to obtain the measurement outcome $a_{j}$, Eq. (26), becomes

$$
\operatorname{Pr}\left[A=a_{j}\right]=\frac{\left\|\left\langle f|V| a_{j}\right\rangle\right\|^{2}\left\|\left\langle a_{j}|U| i\right\rangle\right\|^{2}}{\sum_{j}\left\|\left\langle f|V| a_{j}\right\rangle\right\|^{2}\left\|\left\langle a_{j}|U| i\right\rangle\right\|^{2}} .
$$

This is called the Aharonov-Bergmann-Lebowitz (ABL) formula (6). From the analogous discussion to the above, this measurement is time symmetric. Therefore, describing quantum mechanics by the pre- and post-selected states, $|i\rangle$ and $\langle f|$, is called the "two-state vector formalism".

\subsection{Protective measurement}

In this subsection, we will see the noninvasive quantum measurement for the specific quantum state on the target system. Consider a system of consisting of a target and a probe defined in the Hilbert space $\mathcal{H}_{s} \otimes \mathcal{H}_{p}$. The interaction between the target and the probe is

\footnotetext{
${ }^{8}$ It is, of course, noted that thermodynamics does not have the time symmetric properties from the second law of thermodynamics.
} 
given by

$$
H_{\text {int }}(t)=g(t)(A \otimes \hat{P})
$$

where

$$
\int_{0}^{T} g(t) d t=: g_{0}
$$

The total Hamiltonian is given by

$$
H_{\text {tot }}(t)=H_{s}(t)+H_{p}(t)+H_{\text {int }}(t) .
$$

Here, we suppose that $H_{s}(t)$ has discrete and non-degenerate eigenvalues denoted as $E_{i}(t)$. Its corresponding eigenstate is denoted as $\left|E_{i}(t)\right\rangle$ for any time $t$. Furthermore, we consider the discretized time from the time interval $[0, T]$;

$$
t_{n}=\frac{n}{N} T(n=0,1,2, \ldots, N),
$$

where $N$ is a sufficiently large number. We assume that the initial target state is the energy eigenvalue $\left|E_{i}(t)\right\rangle^{9}$ the initial probe state is denoted as $|\xi(0)\rangle$. Under the adiabatic condition, the compound state for the target and probe systems at time $T$ is given by

$$
\begin{aligned}
|\Phi(T)\rangle:= & \left|E_{i}\left(t_{N}\right)\right\rangle\left\langle E_{i}\left(t_{N}\right)\left|e^{-i \frac{T}{N} H_{\text {tot }}\left(t_{N}\right)}\right| E_{i}\left(t_{N-1}\right)\right\rangle\left\langle E_{i}\left(t_{N-1}\right)\right| e^{-i \frac{T}{N} H_{\text {tot }}\left(t_{N-1}\right)} \ldots \\
& \times\left|E_{i}\left(t_{2}\right)\right\rangle\left\langle E_{i}\left(t_{2}\right)\left|e^{-i \frac{T}{N} H_{\text {tot }}\left(t_{2}\right)}\right| E_{i}\left(t_{1}\right)\right\rangle\left\langle E_{i}\left(t_{1}\right)\left|e^{-i \frac{T}{N} H_{\text {tot }}\left(t_{1}\right)}\right| E_{i}(0)\right\rangle \otimes|\xi(0)\rangle .
\end{aligned}
$$

Applying the Trotter-Suzuki theorem (173; 182), one has

$$
\begin{aligned}
|\Phi(T)\rangle:= & \left|E_{i}\left(t_{N}\right)\right\rangle\left\langle E_{i}\left(t_{N}\right)\left|e^{-i \frac{T}{N} H_{\text {int }}\left(t_{N}\right)}\right| E_{i}\left(t_{N}\right)\right\rangle\left\langle E_{i}\left(t_{N-1}\right)\right| e^{-i \frac{T}{N} H_{\text {int }}\left(t_{N-1}\right)} \ldots \\
& \times\left|E_{i}\left(t_{3}\right)\right\rangle\left\langle E_{i}\left(t_{2}\right)\left|e^{-i \frac{T}{N} H_{\text {int }}\left(t_{2}\right)}\right| E_{i}\left(t_{2}\right)\right\rangle\left\langle E_{i}\left(t_{1}\right)\left|e^{-i \frac{T}{N} H_{\text {int }}\left(t_{1}\right)}\right| E_{i}(1)\right\rangle \otimes|\xi(T)\rangle .
\end{aligned}
$$

By the Taylor expansion with the respect to $N$, the expectation value is

$$
\begin{aligned}
\left\langle E_{i}\left(t_{n}\right)\left|e^{-i \frac{T}{N} g\left(t_{n}\right) A \otimes \hat{P}}\right| E_{i}\left(t_{n}\right)\right\rangle & =1-i \frac{T}{N} g\left(t_{n}\right) \operatorname{Ex}\left[A\left(t_{n}\right)\right] \hat{P}-\frac{1}{2} \frac{T^{2}}{N^{2}} g^{2}\left(t_{n}\right)\left(\operatorname{Ex}\left[A\left(t_{n}\right)\right]\right)^{2} \hat{P}^{2} \\
& -\frac{1}{2} \frac{T^{2}}{N^{2}} g^{2}\left(t_{n}\right) \operatorname{Var}\left[A\left(t_{n}\right)\right] \hat{P}^{2}+O\left(\frac{1}{N^{3}}\right) \\
& \sim e^{-i \frac{T}{N} g\left(t_{n}\right) \operatorname{Ex}\left[A\left(t_{n}\right)\right] \hat{P}}\left(1-\frac{1}{2} \frac{T^{2}}{N^{2}} g^{2}\left(t_{n}\right) \operatorname{Var}\left[A\left(t_{n}\right)\right] \hat{P}^{2}\right) .
\end{aligned}
$$

\footnotetext{
${ }^{9}$ Due to this assumption, it is impossible to apply this to the arbitrary quantum state. Furthermore, while we seemingly need the projective measurement, that is, destructive measurement, for the target system to confirm whether the initial quantum state is in the eigenstates $(145 ; 186)$, they did not apply this to the arbitrary state. For example, if the system is cooled down, we can pickup the ground state of the target Hamiltonian $H_{S}(0)$.
} 
In the limit of $N \rightarrow \infty$, by quadrature by parts, we obtain

$$
\begin{aligned}
|\Phi(T)\rangle \sim\left|E_{i}(T)\right\rangle \exp \left[-i\left(\int_{0}^{T} g(t) \operatorname{Ex}[A(t)] d t\right) \hat{P}\right] \\
\times\left[1-\frac{T}{N}\left(\int_{0}^{T} g^{2}(t) \operatorname{Var}[A(t)] d t\right) \hat{P}^{2}\right]|\xi(T)\rangle+O\left(\frac{1}{N}\right) \\
=\left|E_{i}(T)\right\rangle \exp \left[-i\left(\int_{0}^{T} g(t) \operatorname{Ex}[A(t)] d t\right) \hat{P}\right]|\xi(T)\rangle .
\end{aligned}
$$

Therefore, the shift of the expectation value for the position operator on the probe system is given by

$$
\Delta[Q]=\int_{0}^{T} g(t) \operatorname{Ex}[A(t)] d t
$$

It is emphasized that the quantum state on the target system remains to be the energy eigenstate of $H_{s}$. Therefore, this is called the protective measurement $(5 ; 18)$. It is remarked that the generalized version of the protective measurement in Ref. (19) by the pre- and post-selected states and in Ref. (10) by the meta-stable state.

\subsection{Weak measurement}

From the above discussions, is it possible to combine the above two concepts, i.e., the time-symmetric quantum measurement without destroying the quantum state (189)? This answer is the weak measurement (4). Consider a target system and a probe defined in the Hilbert space $\mathcal{H}_{s} \otimes \mathcal{H}_{p}$. The interaction of the target system and the probe is assumed to be weak and instantaneous,

$$
H_{\text {int }}(t)=g(A \otimes \hat{P}) \delta\left(t-t_{0}\right),
$$

where an observable $A$ is defined in $\mathcal{H}_{s}$, while $\hat{P}$ is the momentum operator of the probe. The time evolution operator becomes $e^{-i g(A \otimes \hat{P})}$. Suppose the probe initial state is $|\xi\rangle$. For the transition from the pre-selected state $|i\rangle$ to the post-selected state $|f\rangle$, the probe wave function becomes $\left|\xi^{\prime}\right\rangle=\left\langle f\left|V e^{-i g(A \otimes \hat{P})} U\right| i\right\rangle|\xi\rangle$, which is in the weak coupling case,

$$
\begin{aligned}
\left|\xi^{\prime}\right\rangle & =\left\langle f\left|V e^{-i g(A \otimes \hat{P})} U\right| i\right\rangle|\xi\rangle=\langle f|V[\mathbf{1}-i g(A \otimes \hat{P})] U| i\rangle|\xi\rangle+O\left(g^{2}\right) \\
& =\langle f|V U| i\rangle-i g\langle f|V A U| i\rangle \otimes \hat{P}|\xi\rangle+O\left(g^{2}\right)=\langle f|V U| i\rangle\left(1-i g\langle A\rangle_{w} \hat{P}\right)|\xi\rangle+O\left(g^{2}\right)
\end{aligned}
$$

where $\langle f|V A U| i\rangle /\langle f|V U| i\rangle=\langle A\rangle_{w}$. Here, the last equation uses the approximation that $g\langle A\rangle_{w} \ll 1^{10}$. We obtain the shifts of the expectation values for the position and momentum operators on the probe as the following theorem:

Theorem 4.1 (Jozsa (85)). We obtain the shifts of the expectation values for the position and momentum operators on the probe after the weak measurement with the post-selection as

$$
\begin{aligned}
& \Delta[\hat{Q}]=g \operatorname{Re}\langle A\rangle_{w}+\left.m g \operatorname{Im}\langle A\rangle_{w} \frac{d \operatorname{Var}[\hat{Q}]}{d t}\right|_{t=t_{0}}, \\
& \Delta[\hat{P}]=2 g \operatorname{Im}\langle A\rangle_{w} \operatorname{Var}[\hat{P}],
\end{aligned}
$$

\footnotetext{
${ }^{10} \mathrm{It}$ is remarked that $\mathrm{Wu}$ and $\mathrm{Li}$ showed the second-order correction of the weak measurement (196). A further analysis was shown in Refs. $(129 ; 132)$.
} 
where

$$
\begin{gathered}
\Delta[\hat{Q}]:=\frac{\left\langle\xi^{\prime}|\hat{Q}| \xi^{\prime}\right\rangle}{\left\langle\xi^{\prime} \mid \xi^{\prime}\right\rangle}-\langle\xi|\hat{Q}| \xi\rangle, \\
\Delta[\hat{P}]:=\frac{\left\langle\xi^{\prime}|\hat{P}| \xi^{\prime}\right\rangle}{\left\langle\xi^{\prime} \mid \xi^{\prime}\right\rangle}-\langle\xi|\hat{P}| \xi\rangle, \\
\operatorname{Var}[\hat{Q}]:=\left\langle\xi\left|\hat{Q}^{2}\right| \xi\right\rangle-(\langle\xi|\hat{Q}| \xi\rangle)^{2}, \\
\operatorname{Var}[\hat{P}]:=\left\langle\xi\left|\hat{P^{2}}\right| \xi\right\rangle-(\langle\xi|\hat{P}| \xi\rangle)^{2} .
\end{gathered}
$$

Here, the probe Hamiltonian is assumed as

$$
\hat{H}=\frac{\hat{P}^{2}}{2 m}+V(Q)
$$

where $V(Q)$ is the potential on the coordinate space.

Proof. For the probe observable $\hat{M}$, we obtain

$$
\begin{aligned}
\frac{\left\langle\xi^{\prime}|\hat{M}| \xi^{\prime}\right\rangle}{\left\langle\xi^{\prime} \mid \xi^{\prime}\right\rangle}= & \frac{\langle\xi|\hat{M}| \xi\rangle-i g\langle A\rangle_{w}\langle\xi|\hat{M}| \hat{P} \mid \xi\rangle+i g \overline{\langle A\rangle_{w}}\langle\xi|\hat{P} \hat{M}| \xi\rangle}{\langle\xi \mid \xi\rangle-i g\langle A\rangle_{w}\langle\xi|\hat{P}| \xi\rangle+i g \overline{\langle A\rangle_{w}}\langle\xi|\hat{P}| \xi\rangle} \\
= & \frac{\langle\xi|\hat{M}| \xi\rangle+i g \operatorname{Re}\langle A\rangle_{w}\langle\xi|[\hat{P}, \hat{M}]| \xi\rangle+g \operatorname{Im}\langle A\rangle_{w}\langle\xi|\{\hat{P}, \hat{M}\}| \xi\rangle}{\langle\xi \mid \xi\rangle+2 g \operatorname{Im}\langle A\rangle_{w}\langle\xi|\hat{P}| \xi\rangle} \\
= & \left(\langle\xi|\hat{M}| \xi\rangle+i g \operatorname{Re}\langle A\rangle_{w}\langle\xi|[\hat{P}, \hat{M}]| \xi\rangle+g \operatorname{Im}\langle A\rangle_{w}\langle\xi|\{\hat{P}, \hat{M}\}| \xi\rangle\right) \\
= & \times\left(1-2 g \operatorname{Im}\langle A\rangle_{w}\langle\xi|\hat{P}| \xi\rangle\right)+O\left(g^{2}\right) \\
& \quad+g \operatorname{Im}|\xi\rangle+i g \operatorname{Re}\langle A\rangle_{w}\langle\xi|[\hat{P}, \hat{M}]| \xi\rangle \\
&
\end{aligned}
$$

If we set $\hat{M}=\hat{P}$, one has

$$
\Delta[\hat{P}]=2 g \operatorname{Im}\langle A\rangle_{w} \operatorname{Var}[\hat{P}] .
$$

If instead we set $\hat{M}=\hat{Q}$, one has

$$
\Delta[\hat{Q}]=g \operatorname{Re}\langle A\rangle_{w}+g \operatorname{Im}\langle A\rangle_{w}(\langle\xi|\{\hat{P}, \hat{Q}\}| \xi\rangle-2 g\langle\xi|\hat{Q}| \xi\rangle\langle\xi|\hat{P}| \xi\rangle)
$$

since $[\hat{P}, \hat{Q}]=-i$. From the Heisenberg equation with the probe Hamiltonian (46), we obtain the Ehrenfest theorem;

$$
\begin{aligned}
i \frac{d}{d t}\langle\xi|\hat{Q}| \xi\rangle & =\langle\xi|[\hat{Q}, \hat{H}]| \xi\rangle=i \frac{\langle\xi|\hat{P}| \xi\rangle}{m} \\
i \frac{d}{d t}\left\langle\xi\left|\hat{Q}^{2}\right| \xi\right\rangle & =\left\langle\xi\left|\left[\hat{Q}^{2}, \hat{H}\right]\right| \xi\right\rangle=i \frac{\langle\xi|\{\hat{P}, \hat{Q}\}| \xi\rangle}{m} .
\end{aligned}
$$


Substituting them into Eq. (49), we derive

$$
\Delta[\hat{Q}]=g \operatorname{Re}\langle A\rangle_{w}+\left.m g \operatorname{Im}\langle A\rangle_{w} \frac{d \operatorname{Var}[\hat{Q}]}{d t}\right|_{t=t_{0}}
$$

since the interaction to the target system is taken at time $t=t_{0}$.

Putting together, we can measure the weak value $\langle A\rangle_{w}$ by observing the shift of the expectation value of the probe both in the coordinate and momentum representations. The shift of the probe position contains the future information up to the post-selected state.

Corollary 4.2. When the probe wavefunction is real-valued in the coordinate representation, Eq. (40) can be reduced to

$$
\Delta[\hat{Q}]=g \operatorname{Re}\langle A\rangle_{w} .
$$

Proof. From the Schrödinger equation in the coordinate representation;

$$
i \frac{\partial}{\partial t} \xi(Q)=\frac{1}{2 m} \frac{\partial^{2}}{\partial Q^{2}} \xi(Q)+V(Q) \xi(Q),
$$

where $\xi(Q) \equiv\langle Q \mid \xi\rangle$, putting $\xi(Q)=R(Q) e^{i S(Q)}$, we obtain the equation for the real part as

$$
\frac{\partial}{\partial t} R(Q)+\frac{\partial}{\partial Q}\left(\frac{R(Q) \frac{\partial}{\partial Q} S(Q)}{m}\right)=0 .
$$

Therefore, if the probe wavefunction is real-valued in the coordinate representation, one has $\frac{\partial}{\partial Q} S(Q)=0$ to obtain $\frac{\partial}{\partial t} R=0$. Therefore, we obtain

$$
\frac{d \operatorname{Var}[\hat{Q}]}{d t}=0
$$

for any time $t$. Vice versa. From this statement, we obtain the desired result from Eq. (40).

It is noted that there are many analyses on the weak measurement, e.g., on the phase space (102), on the finite sample (179), on the counting statics $(26 ; 104)$, on the non-local observable $(32 ; 33)$, and on the complementary observable (197).

Summing up this section, the two-state vector formalism is called if the pre- and post-selected states are prepared and the weak or strong measurement is taken in the von-Neumann type Hamiltonian, $H=g A \hat{P} \delta\left(t-t_{0}\right)$ between the pre- and post-selected states. In the case of the strong measurement, we obtain the expectation value $\operatorname{Ex}(A)$ in the probe. On the other hand, in the case of the weak measurement, we obtain the weak value $\langle A\rangle_{w}$ in the probe.

\section{Weak-value measurement for a qubit system}

In this subsection, we consider the weak measurement in the case that the probe system is a qubit system (195). In general, the interaction Hamiltonian is given by

$$
H_{\text {int }}=g[A \otimes(\vec{v} \cdot \vec{\sigma})] \delta\left(t-t_{0}\right),
$$

where $\vec{v}$ is a unit vector. Expanding the interaction Hamiltonian for the pre- and post-selected states, $|\psi\rangle$ and $|\phi\rangle$, respectively up to the first order for $g$, we obtain the shift of the expectation 
value for $\vec{q} \cdot \vec{\sigma}$ as

$$
\begin{aligned}
\Delta[\vec{q} \cdot \vec{\sigma}]= & \frac{\left\langle\xi^{\prime}|[\vec{q} \cdot \vec{\sigma}]| \xi^{\prime}\right\rangle}{\left\langle\xi^{\prime} \mid \xi^{\prime}\right\rangle}-\langle\xi|[\vec{q} \cdot \vec{\sigma}]| \xi\rangle=g\langle\xi|i[\vec{v} \cdot \vec{\sigma}, \vec{q} \cdot \vec{\sigma}]| \xi\rangle \operatorname{Re}\langle A\rangle_{w} \\
& +g(\langle\xi|\{\vec{v} \cdot \vec{\sigma}, \vec{q} \cdot \vec{\sigma}\}| \xi\rangle-2\langle\xi|\vec{v} \cdot \vec{\sigma}| \xi\rangle\langle\xi|\vec{q} \cdot \vec{\sigma}| \xi\rangle) \operatorname{Im}\langle A\rangle_{w}+O\left(g^{2}\right) \\
= & 2 g\{(\vec{q} \times \vec{v}) \cdot \vec{m}\} \operatorname{Re}\langle A\rangle_{w}+2 g\{\vec{v} \cdot \vec{q}-(\vec{v} \cdot \vec{m})(\vec{q} \cdot \vec{m})\} \operatorname{Im}\langle A\rangle_{w}+O\left(g^{2}\right),
\end{aligned}
$$

where

$$
\begin{aligned}
\left|\xi^{\prime}\right\rangle & =\left\langle\phi\left|e^{-i g[A \otimes(\vec{v} \cdot \vec{\sigma})]}\right| \psi\right\rangle|\xi\rangle, \\
|\zeta\rangle\langle\xi| & =: \frac{1}{2}(\mathbf{1}+\vec{m} \cdot \vec{\sigma}) .
\end{aligned}
$$

Furthermore, the pre- and post-selected states are assumed to be

$$
\begin{aligned}
|\psi\rangle\langle\psi| & =: \frac{1}{2}\left(\mathbf{1}+\vec{r}_{i} \cdot \vec{\sigma}\right), \\
|\phi\rangle\langle\phi| & =: \frac{1}{2}\left(\mathbf{1}+\vec{r}_{f} \cdot \vec{\sigma}\right) .
\end{aligned}
$$

Since the weak value of the observable $\vec{n} \cdot \vec{\sigma}$ is

$$
\langle\vec{n} \cdot \vec{\sigma}\rangle_{w}=\frac{\langle\phi|\vec{n} \cdot \vec{\sigma}| \psi\rangle\langle\psi \mid \phi\rangle}{|\langle\phi \mid \psi\rangle|^{2}}=\vec{n} \cdot \frac{\vec{r}_{i}+\vec{r}_{f}+i\left(\vec{r}_{i} \times \vec{r}_{f}\right)}{1+\vec{r}_{i} \cdot \vec{r}_{f}},
$$

we obtain

$$
\Delta[\vec{q} \cdot \vec{\sigma}]=2 g\{(\vec{q} \times \vec{v}) \cdot \vec{m}\} \frac{\vec{n} \cdot\left(\vec{r}_{i}+\vec{r}_{f}\right)}{1+\vec{r}_{i} \cdot \vec{r}_{f}}+2 g\{\vec{v} \cdot \vec{q}-(\vec{v} \cdot \vec{m})(\vec{q} \cdot \vec{m})\} \frac{\vec{n} \cdot\left(\vec{r}_{i} \times \vec{r}_{f}\right)}{1+\vec{r}_{i} \cdot \vec{r}_{f}}+O\left(g^{2}\right) .
$$

From Eq. (63), we can evaluate the real and imaginary parts of the weak value changing the parameter of the measurement direction $\vec{q}$. This calculation is used in the context of the Hamiltonian estimation (157).

Next, as mentioned before, we emphasize that the weak measurement is only one of the methods to obtain the weak value. There are many other approaches to obtain the weak value, e.g., on changing the probe state $(59 ; 80 ; 103 ; 119)$, and on the entangled probe state $(114)$. Here, we show another method to obtain the weak value in the case that the target and the probe systems are both qubit systems (133).

Let $|\psi\rangle_{s}:=\alpha|0\rangle_{s}+\beta|1\rangle_{s}$ be the pre-selected state for the target system. The initial probe state can described as $|\tilde{\zeta}\rangle_{p}:=\gamma|0\rangle_{p}+\eta|1\rangle_{p}$. It is emphasized that the initial probe state is controllable. Here, the initial states are normalized, that is, $|\alpha|^{2}+|\beta|^{2}=1$ and $|\gamma|^{2}+|\eta|^{2}=1$. Applying the Controlled-NOT (C-NOT) gate, we make a transform of the quantum state for the compound system to

$$
|\psi\rangle_{s} \otimes|\xi\rangle_{p} \stackrel{\mathrm{C}-\mathrm{NOT}}{\longrightarrow}\left|\Psi_{c}\right\rangle:=\left(\alpha \gamma|0\rangle_{s}+\beta \eta|1\rangle_{s}\right)|0\rangle_{p}+\left(\alpha \eta|0\rangle_{s}+\beta \gamma|1\rangle_{s}\right)|1\rangle_{p} .
$$

In the case of $\gamma \sim 1$, we obtain the compound state as

$$
\alpha|0\rangle_{s}|0\rangle_{p}+\beta|1\rangle_{s}|1\rangle_{p}
$$


and similarly, in the case of $\eta \sim 1$, one has

$$
\alpha|0\rangle_{s}|1\rangle_{p}+\beta|1\rangle_{s}|0\rangle_{p}
$$

Those cases can be taken as the standard von Neumann projective measurement. For the post-selected state $|\phi\rangle$, the probability to obtain the measurement outcome $k$ on the probe is

$$
\begin{aligned}
\operatorname{Pr}[k] & :=\frac{\|\left({ }_{s}\left\langle\phi|\otimes p\langle k|) \mid \Psi_{c}\right\rangle \|^{2}\right.}{\sum_{m \in\{0,1\}} \|\left({ }_{s}\left\langle\phi\left|\otimes{ }_{p}\langle m|\right) \mid \Psi_{c}\right\rangle \|^{2}\right.} \\
& =\frac{\left.\mid\left({ }_{s}\langle\phi \mid 0\rangle_{s}\langle 0 \mid \psi\rangle_{s} \gamma+{ }_{s}\langle\phi \mid 1\rangle_{s}\langle 1 \mid \psi\rangle_{s} \eta\right) \delta_{k, 0}+{ }_{s}\langle\phi \mid 0\rangle_{s}\langle 0 \mid \psi\rangle_{s} \eta+{ }_{s}\langle\phi \mid 1\rangle_{s}\langle 1 \mid \psi\rangle_{s} \gamma\right)\left.\delta_{k, 1}\right|^{2}}{\sum_{m \in\{0,1\}}\left\|{ }_{s}\left\langle\phi\left|\otimes{ }_{p}\langle m|\right) \mid \Psi_{c}\right\rangle\right\|^{2}} \\
& =\frac{\left|(\gamma-\eta)_{s}\langle\phi \mid k\rangle_{s}\langle k \mid \psi\rangle_{s}+\eta_{s}\langle\phi \mid \psi\rangle_{s}\right|^{2}}{\left|(\gamma-\eta)_{s}\langle\phi \mid 0\rangle_{s}\langle 0 \mid \psi\rangle_{s}+\eta_{s}\langle\phi \mid \psi\rangle_{s}\right|^{2}+\left|(\gamma-\eta)_{s}\langle\phi \mid 1\rangle_{s}\langle 1 \mid \psi\rangle_{s}+\eta_{s}\langle\phi \mid \psi\rangle_{s}\right|^{2}} \\
& =\frac{\left|(\gamma-\eta)_{\phi}\langle\mid k\rangle_{s}\langle k \mid\rangle_{\psi}^{w}+\eta\right|^{2}}{1-(\gamma-\eta)^{2}\left(1-\left.\sum_{m \in\{0,1\}}{ }_{\phi}\langle\mid m\rangle_{s}\langle m \mid\rangle_{\psi}^{w}\right|^{2}\right)} .
\end{aligned}
$$

Here, in the last line, the parameters $\gamma$ and $\eta$ are assumed to be real. Without the post-selection, the POVM to obtain the measurement outcome $k$ is

$$
E_{k}=\left(\gamma^{2}-\eta^{2}\right)|k\rangle_{s}\langle k|+\eta^{2} .
$$

Here, the coefficient of the first term means that the strength of measurement and the second term is always added. Therefore, we define the quantity to distinguish the probability for the measurement outcome $k$ as

$$
R[k]:=\frac{\operatorname{Pr}[k]-\eta^{2}}{\left(\gamma^{2}-\eta^{2}\right)} .
$$

Putting together Eqs. (67) and (69), we obtain

$$
R[k]=\frac{2 \eta(\gamma-\eta) \operatorname{Re}_{\phi}\langle\mid k\rangle_{s}\langle k \mid\rangle_{\psi}^{w}+(\gamma-\eta)^{2}\left[\left.{ }_{\phi}\langle\mid k\rangle_{s}\langle k \mid\rangle_{\psi}^{w}\right|^{2}+\eta^{2}\left(1-\left|{ }_{\phi}\langle\mid k\rangle_{s}\langle k \mid\rangle_{\psi}^{w}\right|^{2}\right)\right]}{\left(\gamma^{2}-\eta^{2}\right)\left[1-(\gamma-\eta)^{2}\left(1-\sum_{m \in\{0,1\}}\left|{ }_{\phi}\langle\mid m\rangle_{s}\langle m \mid\rangle_{\psi}^{w}\right|^{2}\right)\right]} .
$$

Setting the parameters;

$$
\gamma=\sqrt{\frac{1}{2}+\epsilon}, \quad \eta=\sqrt{\frac{1}{2}-\epsilon}
$$

one has

$$
\begin{aligned}
R[k] & =\frac{(1-\epsilon) \operatorname{Re}_{\phi}\langle\mid k\rangle_{s}\langle k \mid\rangle_{\psi}^{w}+\epsilon\left[\left.\left.\right|_{\phi}\langle\mid k\rangle_{s}\langle k \mid\rangle_{\psi}^{w}\right|^{2}+\left(\frac{1}{2}-\epsilon\right)\left(1-\left.\left.\right|_{\phi}\langle\mid k\rangle_{s}\langle k \mid\rangle_{\psi}^{w}\right|^{2}\right)\right]}{2\left[1-\epsilon^{2}\left(1-\left.\left.\sum_{m \in\{0,1\}}\right|_{\phi}\langle\mid m\rangle_{s}\langle m \mid\rangle_{\psi}^{w}\right|^{2}\right)\right]}+O\left(\epsilon^{2}\right), \\
& =\frac{1}{2} \operatorname{Re}_{\phi}\langle\mid k\rangle_{s}\langle k \mid\rangle_{\psi}^{w}-\frac{\epsilon}{2}\left(\operatorname{Re}_{\phi}\langle\mid k\rangle_{s}\langle k \mid\rangle_{\psi}^{w}-\left.\left.\frac{1}{2}\right|_{\phi}\langle\mid k\rangle_{s}\langle k \mid\rangle_{\psi}^{w}\right|^{2}\right)+O\left(\epsilon^{2}\right) .
\end{aligned}
$$

From Eq. (72), it is possible to obtain the real part of the weak value from the first term and its imaginary part from the second term. Since the first order of the parameter $\epsilon$ is the gradient on 
changing the initial probe state from $|\xi\rangle_{p}=\frac{1}{\sqrt{2}}\left(|0\rangle_{p}+|1\rangle_{p}\right)$, realistically, we can evaluate the imaginary part of the weak value from the gradient of the readout. This method is also used in Ref. (198) on the joint weak value. It is emphasized that the weak value can be experimentally accessible by changing the initial probe state while the interaction is not weak ${ }^{11}$.

\section{Weak values for arbitrary coupling quantum measurement}

We just calculate an arbitrary coupling between the target and the probe systems $(93 ; 120 ; 199)$. Throughout this section, we assume that the desired observable is the projection operator to be denoted as $A^{2}=A$ (153). In the case of the von-Neumann interaction motivated by the original work (4), when the pre- and post-selected states are $|i\rangle$ and $|f\rangle$, respectively, and the probe state is $|\tilde{\xi}\rangle$, the probe state $\left|\xi^{\prime}\right\rangle$ after the interaction given by $H_{\text {int }}=g A \hat{P}$ becomes

$$
\begin{aligned}
\left|\xi^{\prime}\right\rangle & =\left\langle f\left|e^{-i g A \hat{P}}\right| i\right\rangle|\xi\rangle=\left\langle f\left|\left(1+\sum_{k=1}^{\infty} \frac{1}{k !}(-i g A \hat{P})^{k}\right)\right| i\right\rangle|\xi\rangle=\left\langle f\left|\left(1+A \sum_{k=1}^{\infty} \frac{1}{k !}(-i g \hat{P})^{k}\right)\right| i\right\rangle|\xi\rangle \\
& =\left\langle f\left|\left(1-A+A \sum_{k=0}^{\infty} \frac{1}{k !}(-i g \hat{P})^{k}\right)\right| i\right\rangle|\xi\rangle=\left\langle f\left|\left(1-A+A e^{-i g \hat{P}}\right)\right| i\right\rangle|\xi\rangle \\
& =\langle f \mid i\rangle\left(1-\langle A\rangle_{w}+\langle A\rangle_{w} e^{-i g \hat{P}}\right)|\xi\rangle .
\end{aligned}
$$

It is remarked that the desired observable $B$, which satisfies $B^{2}=1(93 ; 120)$, corresponds to $B=2 A-1$. Analogous to Theorem 4.1, we can derive the expectation values of the position and the momentum after the weak measurement. These quantities depends on the weak value $\langle A\rangle_{w}$ and the generating function for the position and the momentum of the initial probe state $|\xi\rangle$.

\section{Weak value with decoherence}

The decoherence results from the coupled system to the environment and leads to the transition from the quantum to classical systems. The general framework of the decoherence was discussed in Sec. 2. In this section, we discuss the analytical expressions for the weak value.

While we directly discuss the weak value with decoherence, the weak value is defined as a complex number. To analogously discuss the density operator formalism, we need the operator associated with the weak value. Therefore, we define a $W$ operator $W(t)$ as

$$
W(t):=U\left(t, t_{i}\right)|i\rangle\langle f| U\left(t_{f}, t\right) .
$$

To facilitate the formal development of the weak value, we introduce the ket state $|\psi(t)\rangle$ and the bra state $\langle\phi(t)|$ as

$$
|\psi(t)\rangle=U\left(t, t_{i}\right)|i\rangle,\langle\phi(t)|=\langle f| U\left(t_{f}, t\right),
$$

so that the expression for the $\mathrm{W}$ operator simplifies to

$$
W(t)=|\psi(t)\rangle\langle\phi(t)| .
$$

\footnotetext{
11 This point seems to be misunderstood. According to Ref. (134), the violation of the Leggett-Garg inequality (100) was shown, but the macroscopic realism cannot be denied since the noninvasive measurability is not realized.
} 
By construction, the two states $|\psi(t)\rangle$ and $\langle\phi(t)|$ satisfy the Schrödinger equations with the same Hamiltonian with the initial and final conditions $\left|\psi\left(t_{i}\right)\right\rangle=|i\rangle$ and $\left\langle\phi\left(t_{f}\right)\right|=\langle f|$. In a sense, $|\psi(t)\rangle$ evolves forward in time while $\langle\phi(t)|$ evolves backward in time. The time reverse of the $\mathrm{W}$ operator (76) is $W^{\dagger}=|\phi(t)\rangle\langle\psi(t)|$. Thus, we can say the $\mathrm{W}$ operator is based on the two-state vector formalism formally described in Refs. $(16 ; 17)$. Even an apparently similar quantity to the W operator (76) was introduced by Reznik and Aharonov (140) in the name of "two-state" with the conceptually different meaning. This is because the W operator acts on a Hilbert space $\mathcal{H}$ but the two-state vector acts on the Hilbert space $\overrightarrow{\mathcal{H}_{1}} \otimes \overleftarrow{\mathcal{H}_{2}}$. Furthermore, while the generalized two-state, which is called a multiple-time state, was introduced (13), this is essentially reduced to the two-state vector formalism. The $\mathrm{W}$ operator gives the weak value of the observable $A^{12}$ as

$$
\langle A\rangle_{W}=\frac{\operatorname{Tr}(W A)}{\operatorname{Tr} W},
$$

in parallel with the expectation value of the observable $A$ by

$$
\operatorname{Ex}[A]=\frac{\operatorname{Tr}(\rho A)}{\operatorname{Tr} \rho}
$$

from Born's rule. Furthermore, the $\mathrm{W}$ operator (74) can be regarded as a special case of a standard purification of the density operator (185). In our opinion, the W operator should be considered on the same footing of the density operator. For a closed system, both satisfy the Schrödinger equation. In a sense, the $W$ operator $W$ is the square root of the density operator since

$$
W(t) W^{\dagger}(t)=|\psi(t)\rangle\left\langle\psi(t)\left|=U\left(t, t_{i}\right)\right| i\right\rangle\langle i| U^{\dagger}\left(t, t_{i}\right),
$$

which describes a state evolving forward in time for a given initial state $\left|\psi\left(t_{i}\right)\right\rangle\left\langle\psi\left(t_{i}\right)|=| i\right\rangle\langle i|$, while

$$
W^{\dagger}(t) W(t)=|\phi(t)\rangle\left\langle\phi(t)\left|=U\left(t_{f}, t\right)\right| f\right\rangle\langle f| U^{\dagger}\left(t_{f}, t\right),
$$

which describes a state evolving backward in time for a given final state $\left|\phi\left(t_{f}\right)\right\rangle\left\langle\phi\left(t_{f}\right)\right|=$ $|f\rangle\langle f|$. The $\mathrm{W}$ operator describes the entire history of the state from the past $\left(t_{i}\right)$ to the future $\left(t_{f}\right)$ and measurement performed at the time $t_{0}$ as we shall see in Appendix 4.3. This description is conceptually different from the conventional one by the time evolution of the density operator. From the viewpoint of geometry, the $\mathrm{W}$ operator can be taken as the Hilbert-Schmidt bundle. The bundle projection is given by

$$
\Pi: W(t) \rightarrow \rho_{i}(t):=W(t) W^{\dagger}(t) .
$$

When the dimension of the Hilbert space is $N: \operatorname{dim} \mathcal{H}=N$, the structure group of this bundle is $U(N)(25$, Sec. 9.3). Therefore, the $\mathrm{W}$ operator has richer information than the density operator formalism as we shall see a typical example of a geometric phase (155). Furthermore, we can express the probability to get the measurement outcome $a_{n} \in A$ due to the ABL formula (28) using the $\mathrm{W}$ operator $W$ as

$$
\operatorname{Pr}\left[A=a_{n}\right]=\frac{\left|\operatorname{Tr} W P_{a_{n}}\right|^{2}}{\sum_{n}\left|\operatorname{Tr} W P_{a_{n}}\right|^{2}}
$$

\footnotetext{
12 While the original notation of the weak values is $\langle A\rangle_{w}$ indicating the "w"eak value of an observable $A$, our notation is motivated by one of which the pre- and post-selected states are explicitly shown as ${ }_{f}\langle A\rangle_{i}^{w}$.
} 
where $A=\sum_{n} a_{n}\left|a_{n}\right\rangle\left\langle a_{n}\right|=: \sum_{n} a_{n} P_{a_{n}}$. This shows the usefulness of the $\mathrm{W}$ operator. Let us discuss a state change in terms of the $\mathrm{W}$ operator and define a map $\mathcal{X}$ as

$$
\mathcal{X}(|\alpha\rangle,|\beta\rangle):=(\mathcal{E} \otimes \mathbf{1})(|\alpha\rangle\langle\beta|),
$$

for an arbitrary $|\alpha\rangle,|\beta\rangle \in \mathcal{H}_{s} \otimes \mathcal{H}_{e}$. Then, we obtain the following theorem on the change of the $\mathrm{W}$ operator such as Theorem 2.1.

Theorem 7.1. For any $W$ operator $W=|\psi(t)\rangle_{s}\langle\phi(t)|$, we expand

$$
|\psi(t)\rangle_{s}=\sum_{m} \psi_{m}\left|\alpha_{m}\right\rangle_{s},|\phi(t)\rangle_{s}=\sum_{m} \phi_{m}\left|\beta_{m}\right\rangle_{s}
$$

with fixed complete orthonormal sets $\left\{\left|\alpha_{m}\right\rangle_{s}\right\}$ and $\left\{\left|\beta_{m}\right\rangle_{s}\right\}$. Then, a change of the $W$ operator can be written as

$$
\mathcal{E}\left(|\psi(t)\rangle_{s}\langle\phi(t)|\right)={ }_{e}\langle\tilde{\psi}(t)|\mathcal{X}(|\alpha\rangle,|\beta\rangle)| \tilde{\phi}(t)\rangle_{e},
$$

where

$$
|\tilde{\psi}(t)\rangle_{e}=\sum_{k} \psi_{k}^{*}\left|\alpha_{k}\right\rangle_{e},|\tilde{\phi}(t)\rangle_{e}=\sum_{k} \phi_{k}^{*}\left|\beta_{k}\right\rangle_{e},
$$

and $|\alpha\rangle$ and $|\beta\rangle$ are maximally entangled states defined by

$$
|\alpha\rangle:=\sum_{m}\left|\alpha_{m}\right\rangle_{s}\left|\alpha_{m}\right\rangle_{e},|\beta\rangle:=\sum_{m}\left|\beta_{m}\right\rangle_{s}\left|\beta_{m}\right\rangle_{e} .
$$

Here, $\left\{\left|\alpha_{m}\right\rangle_{e}\right\}$ and $\left\{\left|\beta_{m}\right\rangle_{e}\right\}$ are complete orthonormal sets corresponding to $\left\{\left|\alpha_{m}\right\rangle_{s}\right\}$ and $\left\{\left|\beta_{m}\right\rangle_{s}\right\}$, respectively.

The proof is completely parallel to that of Theorem 2.1.

Theorem 7.2. For any $W$ operator $W=|\psi(t)\rangle_{s}\langle\phi(t)|$, given the $C P$ map $\mathcal{E}$, the operator-sum representation is written as

$$
\mathcal{E}(W)=\sum_{m} E_{m} W F_{m}^{\dagger}
$$

where $E_{m}$ and $F_{m}$ are the Kraus operators.

It is noted that, in general, $\mathcal{E}(W) \mathcal{E}\left(W^{\dagger}\right) \neq \mathcal{E}(\rho)$ although $\rho=W W^{\dagger}$.

Proof. We take the polar decomposition of the map $X$ to obtain

$$
\mathcal{X}=\mathcal{K} u,
$$

noting that

$$
\mathcal{X} \mathcal{X}^{\dagger}=\mathcal{K} u u^{\dagger} \mathcal{K}=\mathcal{K}^{2} \text {. }
$$

The unitary operator $u$ is well-defined on $\mathcal{H}_{s} \otimes \mathcal{H}_{e}$ because $\mathcal{K}$ defined in Eq. (4) is positive. This is a crucial point to obtain this result (88), which is the operator-sum representation for the quantum operation of the $\mathrm{W}$ operator. From Eq. (10), we can rewrite $\mathcal{X}$ as

$$
\mathcal{X}=\sum_{m}\left|s_{m}\right\rangle\left\langle s_{m}\left|u=\sum_{m}\right| s_{m}\right\rangle\left\langle t_{m}\right|,
$$

where

$$
\left\langle t_{m}\right|=\left\langle s_{m}\right| u .
$$


Similarly to the Kraus operator (16), we define the two operators, $E_{m}$ and $F_{m}^{+}$, as

$$
E_{m}|\psi(t)\rangle_{s}:=e_{e}\left\langle\tilde{\psi}(t) \mid s_{m}\right\rangle, \quad{ }_{s}\langle\phi(t)| F_{m}^{\dagger}:=\left\langle t_{m} \mid \tilde{\phi}(t)\right\rangle_{e},
$$

where $|\tilde{\psi}(t)\rangle_{e}$ and $|\tilde{\phi}(t)\rangle_{e}$ are defined in Eq. (86). Therefore, we obtain the change of the W operator as

$$
\begin{aligned}
\sum_{m} E_{m}|\psi(t)\rangle_{s}\langle\phi(t)| F_{m}^{\dagger} & =\sum_{m}\left\langle\tilde{\psi}(t) \mid s_{m}\right\rangle\left\langle t_{m} \mid \tilde{\phi}(t)\right\rangle_{e}={ }_{e}\langle\tilde{\psi}(t)|\mathcal{X}| \tilde{\phi}(t)\rangle_{e} \\
& =\mathcal{E}\left(|\psi(t)\rangle_{s}\langle\phi(t)|\right),
\end{aligned}
$$

using Theorem 7.1 in the last line. By linearity, we obtain the desired result.

Summing up, we have introduced the $\mathrm{W}$ operator (74) and obtained the general form of the quantum operation of the $\mathrm{W}$ operator (88) in an analogous way to the quantum operation of the density operator assuming the complete positivity of the physical operation. This can be also described from information-theoretical approach (43) to solve the open problem listed in Ref. (13, Sec. XII). However, this geometrical meaning has still been an open problem.

It is well established that the trace preservation, $\operatorname{Tr}(\mathcal{E}(\rho))=\operatorname{Tr} \rho=1$ for all $\rho$, implies that $\sum_{m} E_{m}^{\dagger} E_{m}=1$. As discussed in Eq. (17), the proof goes through as

$$
1=\operatorname{Tr}(\mathcal{E}(\rho))=\operatorname{Tr}\left(\sum_{m} E_{m} \rho E_{m}^{\dagger}\right)=\operatorname{Tr}\left(\sum_{m} E_{m}^{\dagger} E_{m} \rho\right)(\forall \rho) .
$$

This argument for the density operator $\rho=W W^{\dagger}$ applies also for $W^{\dagger} W$ to obtain $\sum_{m} F_{m}^{\dagger} F_{m}=1$ because this is the density operator in the time reversed world in the two-state vector formulation as reviewed in Sec. 4. Therefore, we can express the Kraus operators,

$$
E_{m}=e_{e}\left\langle e_{m}|U| e_{i}\right\rangle_{e}, F_{m}^{+}=e_{e}\left\langle e_{f}|V| e_{m}\right\rangle_{e},
$$

where

$$
U=U\left(t, t_{i}\right), V=U\left(t_{f}, t\right),
$$

are the evolution operators, which act on $\mathcal{H}_{s} \otimes \mathcal{H}_{e} .\left|e_{i}\right\rangle$ and $\left|e_{f}\right\rangle$ are some basis vectors and $\left|e_{m}\right\rangle$ is a complete set of basis vectors with $\sum_{m}\left|e_{m}\right\rangle\left\langle e_{m}\right|=1$. We can compute

$$
\sum_{m} F_{m}^{\dagger} E_{m}=\sum_{m} e_{e}\left\langle e_{f}|V| e_{m}\right\rangle_{e}\left\langle e_{m}|U| e_{i}\right\rangle_{e}=e_{e}\left\langle e_{f}|V U| e_{i}\right\rangle_{e}
$$

The above equality (98) may be interpreted as a decomposition of the history in analogy to the decomposition of unity because

$$
e_{e}\left\langle e_{f}|V U| e_{i}\right\rangle_{e}=e_{e}\left\langle e_{f}|S| e_{i}\right\rangle_{e}=S_{f i}
$$

is the S-matrix element. On this idea, Ojima and Englert have developed the formulation on the S-matrix in the context of the algebraic quantum field theory (123) and the backaction of the Hawking radiation (55), respectively.

\section{Weak measurement with environment}

Let us consider a target system coupled with an environment and a general weak measurement for the compound of the target system and the environment. We assume that 
there is no interaction between the probe and the environment and the same interaction between the target and probe systems (38). The Hamiltonian for the target system and the environment is given by

$$
H=H_{0} \otimes \mathbf{1}_{e}+H_{1}
$$

where $H_{0}$ acts on the target system $\mathcal{H}_{s}$ and the identity operator $\mathbf{1}_{e}$ is for the environment $\mathcal{H}_{e}$, while $H_{1}$ acts on $\mathcal{H}_{s} \otimes \mathcal{H}_{e}$. The evolution operators $U:=U\left(t, t_{i}\right)$ and $V:=U\left(t_{f}, t\right)$ as defined in Eq. (97) can be expressed by

$$
U=U_{0} K\left(t_{0}, t_{i}\right), V=K\left(t_{f}, t_{0}\right) V_{0},
$$

where $U_{0}$ and $V_{0}$ are the evolution operators forward in time and backward in time, respectively, by the target Hamiltonian $H_{0}$. K's are the evolution operators in the interaction picture,

$$
K\left(t_{0}, t_{i}\right)=\mathcal{T} e^{-i \int_{t_{i}}^{t_{0}} d t U_{0}^{+} H_{1} U_{0}}, K\left(t_{f}, t_{0}\right)=\overline{\mathcal{T}} e^{-i \int_{t_{0}}^{t_{f}} d t V_{0} H_{1} V_{0}^{+}},
$$

where $\mathcal{T}$ and $\overline{\mathcal{T}}$ stand for the time-ordering and anti time-ordering products.

Let the initial and final environmental states be $\left|e_{i}\right\rangle$ and $\left|e_{f}\right\rangle$, respectively. The probe state now becomes

$$
\left|\xi^{\prime}\right\rangle=\left\langle f\left|\left\langle e_{f}|V U| e_{i}\right\rangle\right| i\right\rangle\left(\mathbf{1}-g \frac{\left\langle f\left|\left\langle e_{f}|V A U| e_{i}\right\rangle\right| i\right\rangle}{\left\langle f\left|\left\langle e_{f}|V U| e_{i}\right\rangle\right| i\right\rangle} \hat{P}+O\left(g^{2}\right)\right)|\xi\rangle .
$$

Plugging the expressions for $U$ and $V$ into the above, we obtain the probe state as

$$
\left|\xi^{\prime}\right\rangle=N \xi\left(\mathbf{1}-g \frac{\left\langle f\left|\left\langle e_{f}\left|K\left(t_{f}, t_{0}\right) V_{0} A U_{0} K\left(t_{0}, t_{i}\right)\right| e_{i}\right\rangle\right| i\right\rangle}{N} \hat{P}\right)|\xi\rangle+O\left(g^{2}\right),
$$

where $N=\left\langle f\left|\left\langle e_{f}\left|K\left(t_{f}, t_{0}\right) V_{0} U_{0} K\left(t_{0}, t_{i}\right)\right| e_{i}\right\rangle\right| i\right\rangle$ is the normalization factor. We define the dual quantum operation as

$$
\mathcal{E}^{*}(A):=\left\langle e_{f}\left|K\left(t_{f}, t_{0}\right) V_{0} A U_{0} K\left(t_{0}, t_{i}\right)\right| e_{i}\right\rangle=\sum_{m} V_{0} F_{m}^{\dagger} A E_{m} U_{0}
$$

where

$$
\begin{aligned}
& F_{m}^{\dagger}:=V_{0}^{\dagger}\left\langle e_{f}\left|K\left(t_{f}, t_{0}\right)\right| e_{m}\right\rangle V_{0}, \\
& E_{m}:=U_{0}\left\langle e_{m}\left|K\left(t_{0}, t_{i}\right)\right| e_{i}\right\rangle U_{0}^{\dagger}
\end{aligned}
$$

are the Kraus operators. Here, we have inserted the completeness relation $\sum_{m}\left|e_{m}\right\rangle\left\langle e_{m}\right|=1$ with $\left|e_{m}\right\rangle$ being not necessarily orthogonal. The basis $\left|e_{i}\right\rangle$ and $\left|e_{f}\right\rangle$ are the initial and final environmental states, respectively. Thus, we obtain the wave function of the probe as

$$
\begin{aligned}
\left|\xi^{\prime}\right\rangle & =N\left(\mathbf{1}-g \frac{\left\langle f\left|\mathcal{E}^{*}(A)\right| i\right\rangle}{N} \hat{P}\right)|\xi\rangle+O\left(g^{2}\right)=N\left(\mathbf{1}-g \frac{\sum_{m}\left\langle f\left|V_{0} F_{m}^{\dagger} A E_{m} U_{0}\right| i\right\rangle}{\sum_{m}\left\langle f\left|V_{0} F_{m}^{\dagger} E_{m} U_{0}\right| i\right\rangle} \hat{P}\right)|\xi\rangle+O\left(g^{2}\right) \\
& =N\left(\mathbf{1}-g \frac{\operatorname{Tr}\left[A \sum_{m} E_{m} U_{0}|i\rangle\langle f| V_{0} F_{m}^{\dagger}\right]}{\operatorname{Tr}\left[\sum_{m} E_{m} U_{0}|i\rangle\langle f| V_{0} F_{m}^{\dagger}\right]} \hat{P}\right)|\xi\rangle+O\left(g^{2}\right) \\
& =N\left(\mathbf{1}-g \frac{\operatorname{Tr}[\mathcal{E}(W) A]}{\operatorname{Tr}[\mathcal{E}(W)]} \hat{P}\right)|\xi\rangle+O\left(g^{2}\right)=N\left(\mathbf{1}-g\langle A\rangle_{\mathcal{E}(W)} \hat{P}\right)|\xi\rangle+O\left(g^{2}\right)
\end{aligned}
$$


Analogous to Theorem 4.1, the shift of the expectation value of the position operator on the probe is

$$
\Delta[Q]=g \cdot \operatorname{Re}\left[\langle A\rangle_{\mathcal{E}(W)}\right]+\left.m g \cdot \operatorname{Im}\left[\langle A\rangle_{\mathcal{E}(W)}\right] \frac{d \operatorname{Var}[Q]}{d t}\right|_{t=t_{0}} .
$$

From an analogous discussion, we obtain the shift of the expectation value of the momentum operator on the probe as

$$
\Delta[P]=2 g \cdot \operatorname{Var}[P] \cdot \operatorname{Im}\left[\langle A\rangle_{\mathcal{E}(W)}\right] .
$$

Thus, we have shown that the probe shift in the weak measurement is exactly given by the weak value defined by the quantum operation of the $W$ operator due to the environment.

\section{Summary}

We have reviewed that the weak value is defined independent of the weak measurement in the original idea (4) and have explained its properties. Furthermore, to extract the weak value, we have constructed some measurement model to extract the weak value. I hope that the weak value becomes the fundamental quantity to describe quantum mechanics and quantum field theory and has practical advantage in the quantum-mechanical world.

\section{Acknowledgment}

The author acknowledges useful collaborations and discussion with Akio Hosoya, Yuki Susa, and Shu Tanaka. The author thanks Yakir Aharonov, Richard Jozsa, Sandu Popescu, Aephraim Steinberg, and Jeff Tollaksen for useful discussion. The author would like to thank the use of the utilities of Tokyo Institute of Technology and Massachusetts Institute of Technology and many technical and secretary supports. The author is grateful to the financial supports from JSPS Research Fellowships for Young Scientists (No. 21008624), JSPS Excellent Young Researcher Overseas Visit Program, Global Center of Excellence Program "Nanoscience and Quantum Physics" at Tokyo Institute of Technology during his Ph.D study.

\section{References}

[1] J. Åberg and G. Mitchison, J. Math. Phys. 50, 042103 (2009).

[2] G. S. Agarwal and P. K. Pathak, Phys. Rev. A 75, 032108 (2007).

[3] Y. Aharonov, D. Z. Albert, A. Casher, and L. Vaidman, Phys. Lett. A 124, 199 (1987).

[4] Y. Aharonov, D. Z. Albert, and L. Vaidman, Phys. Rev. Lett. 60, 1351 (1988).

[5] Y. Aharonov, J. Anandan, and L. Vaidman, Phys. Rev. A 47, 4616 (1993).

[6] Y. Aharonov, P. G. Bergmann, and J. L. Lebowitz, Phys. Rev. 134, B1410 (1964).

[7] Y. Aharonov and A. Botero, Phys. Rev. A 72, 052111 (2005).

[8] Y. Aharonov, A. Botero, S. Popescu, B. Reznik, and J. Tollaksen, Phys. Lett. A 301, 130 (2002).

[9] Y. Aharonov and E. Y. Gruss, arXiv:quant-ph/0507269.

[10] Y. Aharonov, S. Massar, S. Popescu, J. Tollaksen, and L. Vaidman, Phys. Rev. Lett. 77, 983 (1996).

[11] Y. Aharonov, S. Popescu, D. Rohrlich, and L. Vaidman, Phys. Rev. A 48, 4084 (1993).

[12] Y. Aharonov, S. Popescu, and J. Tollaksen, Physics Today 63, 27 (2010).

[13] Y. Aharonov, S. Popescu, J. Tollaksen, and L. Vaidman, Phys. Rev. A 79, 052110 (2009).

[14] Y. Aharonov and D. Rohrlich, Quantum Paradoxes (Wiley-VCH, Weibheim, 2005). 
[15] Y. Aharonov and J. Tollaksen, in Visions of Discovery: New Light on Physics Cosmology and Consciousness, edited by R. Y. Chiao, M. L. Cohen, A. J. Leggett, W. D. Phillips, and C. L. Harper, Jr. (Cambridge University Press, Cambridge, 2011), p. 105.

[16] Y. Aharonov and L. Vaidman, Phys. Rev. A 41, 11 (1990).

[17] Y. Aharonov and L. Vaidman, J. Phys. A 24, 2315 (1991).

[18] Y. Aharonov and L. Vaidman, Phys. Lett. A 178, 38 (1993).

[19] Y. Aharonov and L. Vaidman, Ann. N.Y. Acad. Sci. 755, 361 (1995).

[20] Y. Aharonov and L. Vaidman, in Time in Quantum Mechanics, Vol. 1, edited by J. G. Muga, R. Sala Mayato, and I. L. Egusquiza (Springer, Berlin Heidelberg, 2008) p. 399.

[21] S. E. Ahnert and M. C. Payne, Phys. Rev. A 69, 042103 (2004).

[22] J. Anandan, Found. Phys. Lett. 15, 415 (2002).

[23] S. Ashhab and F. Nori, arXiv:0907.4823.

[24] A. Bednorz and W. Belzig, Phys. Rev. Lett. 105, 106803 (2010).

[25] I. Bengtsson and K. Życzkowski, Geometry of Quantum States (Cambridge University Press, Cambridge, 2006).

[26] M. V. Berry and P. Shukla, J. Phys. A 43, 354024 (2010).

[27] M. V. Berry, M. R. Dennis, B. McRoberts, and P. Shukla, J. Phys. A 44, 205301 (2011).

[28] M. V. Berry, N. Brunner, S. Popescu, and P. Shukla, J. Phys. A 44, 205301 (2011).

[29] A. Botero and B. Reznik, Phys. Rev. A 61, 050301 (2000)(R).

[30] A. Botero, "Entanglement, Postselection and Precise Inferences in Joint Measurements of Incompatible Observables", in International Conference on Quantum Information, (Optical Society of America, 2008), paper JMB68.

[31] V. B. Braginsky and F. Y. Khalili, Quantum Measurement (Cambridge University Press, Cambridge, 1992).

[32] A. Brodutsch, Master Thesis at Tel-Aviv University (2008), arXiv:0811.1706.

[33] A. Brodutsch and L. Vaidman, J. Phys.: Conf. Ser. 174, 012004 (2009).

[34] R. Brout, S. Massar, R. Parentani, and Ph. Spindel, Phys. Rep. 260, 329 (1995).

[35] T. Brun, L. Diósi, and W. T. Strunz, Phys. Rev. A 77, 032101 (2008).

[36] N. Brunner, A. Acín, D. Collins, N. Gisin, and V. Scarani, Phys. Rev. Lett. 91, 180402 (2003).

[37] N. Brunner, V. Scarani, M. Wegmüller, M. Legré, and N. Gisin, Phys. Rev. Lett. 93, 203902 (2004).

[38] N. Brunner and C. Simon, Phys. Rev. Lett. 105, 010405 (2010).

[39] P. Busch, Found. Phys. 20, 33 (1990).

[40] P. Busch, P. Mittelstaedt, and P. J. Lahti, Quantum Theory of Measurement (Springer-Verlag, Berlin, 1991).

[41] R. M. Camacho, P. B. Dixon, R. T. Glasser, A. N. Jordan, and J. C. Howell, Phys. Rev. Lett. 102, 013902 (2009).

[42] D. Campo and R. Parentani, Phys. Rev. D 70, 105020 (2004).

[43] G. Chiribella, G. M. D’Ariano, and P. Perinotti, Phys. Rev. A 80, 022339 (2009).

[44] Y.-W. Cho, H.-T. Lim, Y.-S. Ra, and Y.-H. Kim, New. J. Phys. 12, 023036 (2010).

[45] E. B. Davies and J. T. Lewis, Commun. Math. Phys. 17, 239 (1970).

[46] P. C. W. Davies, Phys. Rev. A 79, 032103 (2009).

[47] P. A. M. Dirac, Proc. Roy. Soc. Lond. A 180, 1 (1942).

[48] P. B. Dixon, D. J. Starling, A. N. Jordan, and J. C. Howell, Phys. Rev. Lett. 102, 173601 (2009).

[49] J. Dressel, S. Agarwal, and A. N. Jordan, Phys. Rev. Lett. 104, 240401 (2010).

[50] J. Dressel, C. J. Broadbent, J. C. Howell, and A. N. Jordan, Phys. Rev. Lett. 106, 040402 (2011). 
[51] J. Dressel and A. N. Jordan, arXiv:1110.0418.

[52] I. M. Duck, P. M. Stevenson, E. C. G. Sudarshan, Phys. Rev. D 40, 2112 (1989).

[53] G. F. R. Ellis and T. Rothman, Int. J. Theor. Phys. 49, 998 (2010).

[54] F. Englert, arXiv:gr-qc/9502039.

[55] F. Englert and Ph. Spindel, JHEP 12, 065 (2010).

[56] A. Feizpour, X. Xing, and A. Steinberg, Phys. Rev. Lett. 107, 133603 (2011).

[57] R. P. Feynman, Int. J. Theor. Phys. 21, 467 (1982).

[58] J. L. Garretson, H. M. Wiseman, D. T. Pope, and D. T. Pegg, J. Opt. B 6, S506 (2004).

[59] T. Geszti, Phys. Rev. A 81, 044102 (2010).

[60] R. Golub and R. Gähler, Phys. Lett. A 136, 178 (1989).

[61] M. A. de Gosson and S. M. de Gosson, arXiv:1109.3665.

[62] E. Y. Gruss, A Suggestion for a Teleological Interpretation of Quantum Mechanics, as Master Thesis at The Hebrew University of Jerusalem (2000), arXiv:quant-ph/0006070.

[63] E. Haapasalo, P. Lahti, and J. Schultz, Phys. Rev. A 84, 052107 (2011).

[64] L. Hardy, Phys. Rev. Lett. 68, 2981 (1992).

[65] H. F. Hofmann, Phys. Rev. A 62, 022103 (2000).

[66] H. F. Hofmann, J. Phys. A 42, 275304 (2009).

[67] H. F. Hofmann, Phys. Rev. A 81, 012103 (2010).

[68] H. F. Hofmann, Phys. Rev. A 83, 022106 (2011).

[69] H. F. Hofmann, New J. Phys. 13, 103009 (2011).

[70] H. F. Hofmann, AIP Conf. Proc. 1363, 125 (2011).

[71] H. F. Hofmann, arXiv:0911.0071.

[72] H. F. Hofmann, arXiv:1005.0654.

[73] H. F. Hofmann, M. E. Goggin, M. P. Almeida, and M. Barbieri, arXiv:1107.4735.

[74] A. Hosoya and M. Koga, J. Phys. A 44, 415303 (2011).

[75] A. Hosoya and Y. Shikano, J. Phys. A 43, 385307 (2010).

[76] O. Hosten and P. Kwiat, Science 319, 787 (2008).

[77] J. C. Howell, D. J. Starling, P. B. Dixon, P. K. Vudyasetu, and A. N. Jordan, Phys. Rev. A 81, 033813 (2010).

[78] W. Hu, Found. Phys. 20, 447 (1990).

[79] M. Iinuma, Y. Suzuki, G. Taguchi, Y. Kadoya, and H. F. Hofmann, New J. Phys. 13, 033041 (2011).

[80] L. M. Johansen, Phys. Rev. Lett. 93, 120402 (2004).

[81] L. M. Johansen, Phys. Lett. A 322, 298 (2004).

[82] L. M. Johansen, Phys. Lett. A 366, 374 (2007).

[83] A. N. Jordan and A. N. Korotkov, Contemp. Phys. 51,125 (2010).

[84] A. N. Jordan, B. Trauzettel, and G. Burkard, Phys. Rev. B 76, 155324 (2007).

[85] R. Jozsa, Phys. Rev. A 76, 044103 (2007).

[86] S. Kagami, Y. Shikano, and K. Asahi, Physica E 43, 761 (2011).

[87] R. E. Kastner, Stud. Hist. Philos. M. P. 35, 57 (2004).

[88] J. M. Knight and L. Vaidman, Phys. Lett. A 143, 357 (1990).

[89] H. Kobayashi, S. Tamate, T. Nakanishi, K. Sugiyama, and M. Kitano, Phys. Rev. A 81, 012104 (2010).

[90] H. Kobayashi, S. Tamate, T. Nakanishi, K. Sugiyama, and M. Kitano, J. Phys. Soc. Jpn. 80, 034401 (2011).

[91] S. Kocsis, B. Braverman, S. Ravets, M. J. Stevens, R. P. Mirin, L. K. Shalm, and A. M. Steinberg, Science 332, 1170 (2011).

[92] A. G. Kofman, S. Ashhab, and F. Nori, arXiv:1109.6315.

[93] T. Koike and S. Tanaka, Phys. Rev. A 84, 062106 (2011). 
[94] A. N. Korotkov and A. N. Jordan, Phys. Rev. Lett. 97, 166805 (2006).

[95] K. Kraus, Ann. Phys. 64, 311 (1971).

[96] C. M. Krowne, Phys. Lett. A 373, 466 (2009).

[97] A. Palacios-Laloy, F. Mallet, F. Nguyen, P. Bertet, D. Vion, D. Esteve, and A. Korotkov, Nat. Phys. 6, 442 (2010).

[98] C. R. Leavens, Found. Phys. 35, 469 (2005).

[99] C. R. Leavens, I. P. Gimenez, D. Alonso, and R. Sala Mayato, Phys. Lett. A 359, 416 (2006).

[100] A. J. Leggett and A. Garg, Phys. Rev. Lett. 54, 857 (1985).

[101] C.-F. Li, X.-Y. Xu, J.-S. Tang, J.-S. Xu, and G.-C. Guo, Phys. Rev. A 83, 044102 (2011).

[102] A. C. Lobo and C. A. Ribeiro, Phys. Rev. A 80, 012112 (2009).

[103] A. Di Lorenzo and J. C. Egues, Phys. Rev. A 77, 042108 (2008).

[104] A. Di Lorenzo, arXiv:1109.4183.

[105] G. Lüders, Ann. Physik 8, 322 (1951).

[106] A. P. Lund and H. M. Wiseman, New J. Phys. 12, 093011 (2010).

[107] J. S. Lundeen and A. M. Steinberg, Phys. Rev. Lett. 102, 020404 (2009).

[108] J. S. Lundeen and C. Bamber, arXiv:1110.0727.

[109] J. S. Lundeen, B. Sutherland, A. Patel, C. Stewart, and C. Bamber, Nature 474, 188 (2011).

[110] S. Marcovitch and B. Reznik, arXiv:1103.2557.

[111] S. Massar and S. Popescu, Phys. Rev. A 84, 052106 (2011).

[112] D. Menzies and S. Croke, arXiv:0903.4181.

[113] D. Menzies and N. Korolkova, Phys. Rev. A 76, 062310 (2007).

[114] D. Menzies and N. Korolkova, Phys. Rev. A 77, 062105 (2008).

[115] D. J. Miller, in Wagga 2009: 33rd Annual Condensed Matter and Material Meeting, paper WP10.

[116] R. Mir, J. S. Lundeen, H. M. Mitchell, A. M. Steinberg, J. L. Garretson, and H. M. Wiseman, New J. Phys. 9, 287 (2007).

[117] G. Mitchison, Phys. Rev. A 77, 052102 (2008).

[118] G. Mitchison, R. Jozsa, and S. Popescu, Phys. Rev. A 76, 062105 (2007).

[119] K. Nakamura, J. Phys. Soc. Jpn. 79, 125003 (2010).

[120] K. Nakamura, A. Nishizawa, M.-K. Fujimoto, arXiv:1108.2114.

[121] J. von Neumann, Mathematische Grundlagen der Quantumechanik (Springer, Berlin, 1932), [ Eng. trans. by R. T. Beyer, Mathematical foundations of quantum mechanics (Princeton University Press, Princeton, 1955). ]

[122] M. A. Nielsen and I. L. Chuang, Quantum Computation and Quantum Information (Cambridge University Press, Cambridge, 2000).

[123] I. Ojima, in Quantum Bio-Informatics III, From Quantum Information and Bio-Informatics, edited by L. Accardi, W. Freudenberg, and M. Ohya (World Scientific, Singapore, 2010) p. 277.

[124] M. Onoda, S. Murakami, and N. Nagaosa, Phys. Rev. Lett. 93, 083901 (2004).

[125] OPERA Collaboration, T. Adam et al., arXiv:1109.4897.

[126] O. Oreshukov and T. A. Brun, Phys. Rev. Lett. 95, 110409 (2005).

[127] M. Ozawa, J. Math. Phys. 25, 79 (1984).

[128] M. Ozawa, in Squeezed and Nonclassical Light, edited by P. Tombesi and E. R. Pike, (Plenum, New York, 1989), p. 263.

[129] A. K. Pan and A. Matzkin, arXiv:1109.6824.

[130] A. D. Parks, J. Phys. A 43, 035305 (2010).

[131] A. D. Parks, D. W. Cullin, and D. C. Stoudt, Proc. R. Soc. Lond. A 454, 2997 (1998). 
[132] A. D. Parks and J. E. Gray, Phys. Rev. A 84, 012116 (2011).

[133] G. J. Pryde, J. L. O’Brien, A. G. White, T. C. Ralph, and H. M. Wiseman, Phys. Rev. Lett. 94, 220405 (2005).

[134] M. E. Goggin, M. P. Almeida, M. Barbieri, B. P. Lanyon, J. L. O’Brien, A. G. White, and G. J. Pryde, Proc. Natl. Acad. Sci. U. S. A. 108, 1256 (2011).

[135] A. Ranfagni, P. Fabeni, G. P. Pazzi, and D. Mugnai, Phys. Rev. E 48, 1453 (1993).

[136] A. Ranfagni, D. Mugnai, and R. Ruggeri, J. Appl. Phys. 95, 2217 (2004).

[137] T. Ravon and L. Vaidman, J. Phys. A 40, 2873 (2007).

[138] M. Rédei and S. J. Summers, Stud. Hist. Philos. Sci. B 38, 390 (2007).

[139] K. J. Resch, J. S. Lundeen, and A. M. Steinberg, Phys. Lett. A 324, 125 (2004).

[140] B. Reznik and Y. Aharonov, Phys. Rev. A 52, 2538 (1995).

[141] N. W. M. Ritchie, J. G. Story, and R. G. Hulet, Phys. Rev. Lett. 66, 1107 (1991).

[142] D. Rohrlich and Y. Aharonov, Phys. Rev. A 66, 042102 (2002).

[143] A. Romito and Y. Gefen, Physica E 42, 343 (2010).

[144] A. Romito, Y. Gefen, and Y. M. Blanter, Phys. Rev. Lett. 100, 056801 (2008).

[145] C. Rovelli, Phys. Rev. A 50, 2788 (1994).

[146] J. Ruseckas and B. Kaulakys, Phys. Rev. A 66, 052106 (2002).

[147] J. Ruseckas and B. Kaulakys, Lith. J. Phys. 44, 161 (2004).

[148] T. Sagawa, arXiv:0901.4212.

[149] L. K. Shalm, S. Kocsis, S. Ravets, B. Braverman, M. J. Stevens, R. P. Mirin, and A. M. Steinberg, “Observation of Bohmian Trajectories of a Single Photon Using Weak Measurements," in Quantum Electronics and Laser Science Conference, OSA Technical Digest (CD) (Optical Society of America, 2010), paper QThK7.

[150] A. Shapere and F. Wilczek, Geometric Phases in Physics (World Scientific, Singapore, 1988).

[151] Y. Shikano, Weak Values in Quantum Measurement Theory — Concepts and Applications -, as Master Thesis at Tokyo Institute of Technology (2009).

[152] Y. Shikano, Time in Weak Values and Discrete Time Quantum Walk as Ph. D. Dissertation at Tokyo Institute of Technology (2011).

[153] Y. Shikano, unpublished (2010).

[154] Y. Shikano and A. Hosoya, J. Math. Phys. 49, 052104 (2008).

[155] Y. Shikano and A. Hosoya, J. Phys. A 42, 025304 (2010).

[156] Y. Shikano, S. Kagami, S. Tanaka, and A. Hosoya, AIP Conf. Proc. 1363, 177 (2011).

[157] Y. Shikano and S. Tanaka, Europhys. Lett. 96, 40002 (2011).

[158] V. Shpitalnik, Y. Gefen, and A. Romito, Phys. Rev. Lett. 101, 226802 (2008).

[159] C. Simon and E. S. Polzik, Phys. Rev. A 83, 040101 (2011)(R).

[160] E. Sjöqvist, Phys. Lett. A 359, 187 (2006).

[161] E. Sjöqvist and J. Åberg, Phys. Lett. A 354, 396 (2006).

[162] D. Sokolovski, A. Z. Msezane, and V. R. Shaginyan, Phys. Rev. A 71, 064103 (2005).

[163] D. Sokolovski, Phys. Rev. A 76, 042125 (2007).

[164] D. R. Solli, C. F. McCormick, R. Y. Chiao, S. Popescu, and J. M. Hickmann, Phys. Rev. Lett. 92, 043601 (2004).

[165] S. E. Spence and A. D. Parks, to appear in Found. Phys., arXiv:1010.3289.

[166] D. J. Starling, P. B. Dixon, A. N. Jordan, and J. C. Howell, Phys. Rev. A 80, 041803 (2009).

[167] D. J. Starling, P. B. Dixon, A. N. Jordan, and J. C. Howell, Phys. Rev. A 82, 063822 (2010).

[168] D. J. Starling, P. B. Dixon, N. S. Williams, A. N. Jordan, and J. C. Howell, Phys. Rev. A 82, 011802 (2010). 
[169] D. J. Starling, P. B. Dixon, N. S. Williams, A. N. Jordan, and J. C. Howell, "Near Quantum Limited Optical Phase Measurements on a Dark Fringe," in Frontiers in Optics, OSA Technical Digest (CD) (Optical Society of America, 2010), paper FThD4.

[170] A. M. Steinberg, Phys. Rev. Lett. 74, 2405 (1995).

[171] A. M. Steinberg, Phys. Rev. A 52, 32 (1995).

[172] A. M. Steinberg, private communication (2011).

[173] M. Suzuki, Prog. Theor. Phys. 56, 1454 (1976).

[174] S. Tamate, H. Kobayashi, T. Nakanishi, K. Sugiyama, and M. Kitano, New J. Phys. 11, 093025 (2009).

[175] A. Tanaka, Phys. Lett. A 297307 (2002).

[176] S. Tanimura, arXiv:1110.1790.

[177] H. Tomita, arXiv:1109.4302.

[178] J. Tollaksen, J. Phys.: Conf. Ser. 70, 012014 (2007).

[179] J. Tollaksen, J. Phys.: Conf. Ser. 70, 012015 (2007).

[180] J. Tollaksen, J. Phys.: Conf. Ser. 70, 012016 (2007).

[181] J. Tollaksen, Y. Aharonov, A. Casher, T. Kaufherr, and S. Nussinov, New J. Phys. 12, 013023 (2010).

[182] H. F. Trotter, Proc. Amer. Math. Soc. 10, 545 (1959).

[183] M. Tsang, Phys. Rev. A 81, 013824 (2010).

[184] M. D. Turner, C. A. Hagedorn, S. Schlamminger, and J. H. Gundlach, Opt. Lett. 36, 1479 (2011).

[185] A. Uhlmann, Rep. Math. Phys. 24, 229 (1986).

[186] W. G. Unruh, Phys. Rev. A 50, 882 (1994).

[187] K. Urbanowski, Cent. Eur. J. Phys. 7,696 (2009).

[188] L. Vaidman, Found. Phys. 26, 895 (1996).

[189] L. Vaidman, arXiv:quant-ph/9607023.

[190] M. S. Wang, Phys. Rev. Lett. 79, 3319 (1997).

[191] N. S. Williams and A. N. Jordan, Phys. Rev. Lett. 100, 026804 (2008).

[192] H. M. Wiseman, Phys. Rev. A 65, 032111 (2002).

[193] H. M. Wiseman, New J. Phys. 9, 165 (2007).

[194] H. M. Wiseman and G. Milburn, Quantum Measurement and Control (Cambridge University Press, Cambridge, 2010).

[195] S. Wu and K. Kølmer, Phys. Lett. A 374, 34 (2009).

[196] S. Wu and Y. Li, Phys. Rev. A 83, 052106 (2011).

[197] S. Wu and M. Życzkowski, arXiv:1106.4607.

[198] K. Yokota, T. Yamamoto, M. Koashi, and N. Imoto, New J. Phys. 11, 033011 (2009).

[199] X. Zhu, Y. Zhang, S. Pang, C. Qiao, Q. Liu, and S. Wu, Phys. Rev. A 84, 052111 (2011).

[200] O. Zilberberg, A. Romito, and Y. Gefen, Phys. Rev. Lett. 106, 080405 (2011). 


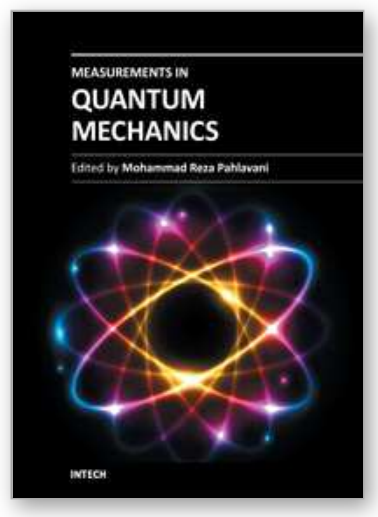

\author{
Measurements in Quantum Mechanics \\ Edited by Prof. Mohammad Reza Pahlavani
}

ISBN 978-953-51-0058-4

Hard cover, 348 pages

Publisher InTech

Published online 22, February, 2012

Published in print edition February, 2012

Perhaps quantum mechanics is viewed as the most remarkable development in 20th century physics. Each successful theory is exclusively concerned about "results of measurement". Quantum mechanics point of view is completely different from classical physics in measurement, because in microscopic world of quantum mechanics, a direct measurement as classical form is impossible. Therefore, over the years of developments of quantum mechanics, always challenging part of quantum mechanics lies in measurements. This book has been written by an international invited group of authors and it is created to clarify different interpretation about measurement in quantum mechanics.

\title{
How to reference
}

In order to correctly reference this scholarly work, feel free to copy and paste the following:

Yutaka Shikano (2012). Theory of "Weak Value" and Quantum Mechanical Measurements, Measurements in Quantum Mechanics, Prof. Mohammad Reza Pahlavani (Ed.), ISBN: 978-953-51-0058-4, InTech, Available from: http://www.intechopen.com/books/measurements-in-quantum-mechanics/theory-of-weak-value-andquantum-mechanical-measurement

\section{INTECH}

open science | open minds

\section{InTech Europe}

University Campus STeP Ri

Slavka Krautzeka 83/A

51000 Rijeka, Croatia

Phone: +385 (51) 770447

Fax: +385 (51) 686166

www.intechopen.com

\section{InTech China}

Unit 405, Office Block, Hotel Equatorial Shanghai

No.65, Yan An Road (West), Shanghai, 200040, China

中国上海市延安西路65号上海国际贵都大饭店办公楼405单元

Phone: +86-21-62489820

Fax: +86-21-62489821 
(C) 2012 The Author(s). Licensee IntechOpen. This is an open access article distributed under the terms of the Creative Commons Attribution 3.0 License, which permits unrestricted use, distribution, and reproduction in any medium, provided the original work is properly cited. 